\title{
Using deconvolution to improve the metrological performance of the grid method
}

\author{
Michel Grédiac ${ }^{1 \dagger}$, Frédéric Sur $^{2}$, Claudiu Badulescu ${ }^{3}$, Jean-Denis Mathias $^{4}$ \\ ${ }^{1}$ Clermont Université, Université Blaise Pascal, Institut Pascal, UMR CNRS 6602 \\ BP 10448, 63000 Clermont-Ferrand, FRANCE \\ ${ }^{2}$ Laboratoire Lorrain de Recherche en Informatique et ses Applications, UMR CNRS 7503 \\ Université de Lorraine, CNRS, INRIA projet Magrit, Campus Scientifique, BP 239 \\ 54506 Vandoeuvre-lès-Nancy Cedex, FRANCE \\ ${ }^{3}$ Laboratoire Brestois de Mécanique et des Systèmes \\ ENSTA Bretagne, 2 rue François Verny, 29806 Brest Cedex 9, FRANCE \\ ${ }^{4}$ IRSTEA, Laboratoire d'Ingénierie pour les Systèmes Complexes \\ Campus universitaire des Cézeaux, 24 avenue des Landais, BP50085, 63172 Aubière Cedex, FRANCE \\ ${ }^{\dagger}$ corresponding author, tel: +3347328 80 77, fax: +3347328 8027 , \\ michel.grediac@univ-bpclermont.fr
}

\begin{abstract}
The use of various deconvolution techniques to enhance strain maps obtained with the grid method is addressed in this study. Since phase derivative maps obtained with the grid method can be approximated by their actual counterparts convolved by the envelope of the kernel used to extract phases and phase derivatives, non-blind restoration techniques can be used to perform deconvolution. Six deconvolution techniques are presented and employed to restore a synthetic phase derivative map, namely direct deconvolution, regularized deconvolution, Richardson-Lucy algorithm and Wiener filtering, the last two with two variants concerning their practical implementations. Obtained results show that the noise that corrupts the grid images must be thoroughly taken into account to limit its effect on the deconvolved strain maps. The difficulty here is that the noise on the grid image yields a spatially correlated noise on the strain maps. In particular, numerical experiments on synthetic data show that direct and regularized deconvolutions are unstable when noisy data are processed. The same remark holds when Wiener filtering is employed without taking into account noise autocorrelation. On the other hand, the Richardson-Lucy algorithm and Wiener filtering with noise autocorrelation provide deconvolved maps where the impact of noise remains controlled within a certain limit. It is also observed that the last technique outperforms the Richardson-Lucy algorithm. Two short examples of actual strain fields restoration are finally shown. They deal with asphalt and shape memory alloy specimens. The benefits and limitations of deconvolution are presented and discussed in these two cases. The main conclusion is that strain maps are correctly deconvolved when the signal-to-noise ratio is high and that actual noise in the actual strain maps must be more specifically characterized than in the current study to address higher noise levels with Wiener filtering.
\end{abstract}

Keywords: deconvolution, grid method, strain measurement 


\section{Introduction}

Full-field measurement techniques are now wide spread in the experimental mechanics community. One of the reasons is their ability to measure heterogeneous states of strain, thus enabling to detect localized events that often occur in specimens under test but whose location is not known a priori. Classic transducers such as strain gauges only give local information, so they are not well suited to reach this goal despite a resolution generally recognized to be better than that of full-field measurement techniques. Assessing the real metrological performances of full-field measurement techniques remains an open problem, as illustrated by the increasing number of papers on this topic. They mainly deal with digital image correlation for which both the surface marking $[1,2,3]$ and the algorithm which are used $[4,5]$ constitute an issue. One of the difficulties comes from the fact that several parameters influence these performances, some of them being not really intrinsic, but extrinsic such as lighting or image contrast for instance [6]. Another reason is the fact that metrological performances depend, among others, on two conflicting concepts: resolution and spatial resolution. The first one is defined here by the smallest strain or displacement that can be detected, the second one by the smallest distance between independent measurements. It is well known that the better the resolution, the worst the spatial resolution. In addition, displacement is generally the physical quantity which is provided by most techniques but strain is often the desired physical quantity for material characterization purposes. The reason is that this quantity is directly involved in constitutive equations of engineering materials. Since displacement maps are generally noisy, they are smoothed using various strategies prior to the differentiation necessary to get the strain components $[7,8]$. Hence the number of independent parameters influencing the final result is in general significant and changing one of the parameters has a direct impact on the obtained strain value. This does not help to give a clear idea on the actual metrological performances of full-field measurement techniques if strain components are to be considered instead of displacements. Finally, it must be pointed out that finding a reference state of strain is also an issue. Specific testing devices or procedures have been proposed in the literature [9, 10] but synthetic images are used in most cases as in [4] for digital image correlation or in [11] for techniques for which fringes must be processed.

This paper is devoted to a specific problem: the actual strain value determination in case of localized phenomena which occur in many situations, especially when heterogeneous materials are tested since the different phases may potentially exhibit various mechanical responses. "Localized" means here that the dimensions of the phenomenon to be observed and for which actual strain components are to be determined are similar to, or lower than the size of the region involved in the calculation of the strain components by local image processing, in other words that the strain gradient is significant.

The measurement method used herein is the grid method, which consists first in depositing a regular bi-directional grid, in taking images of this grid and finally processing these images to retrieve the in-plane strain components. The drawback of this technique is that a regular marking (more precisely a pseudo-regular since printing the grids induces some defects) of the surface under investigation must be obtained by any means, by engraving, transferring or bonding for instance. This surface preparation is in general more difficult than considering a random marking. The advantage is however that a regular marking is much easier to duplicate or to control than a random one. Moreover, a regular marking enables one to use techniques based on the Fourier transform, for which a broad literature is available, especially in image processing [12]

Processing grid images to retrieve strains consists first in taking pictures of the grid before and after the load is applied, and to quantify the changes in the images due to deformation. The 
"classic" procedure for processing these grids consists in extracting the phase using a windowed Fourier transform because phase change is proportional to displacement [13]. The obtained displacement field is then smoothed and differentiated using a suitable strategy to deduce the strain field $[14,8,15]$ for instance. A consequence of this "classic" procedure is that several parameters influence the result, and thus the final metrological performance in terms of quality of the strain measurement. On the contrary, retrieving directly the phase and differentiating it directly without any smoothing, or retrieving the phase derivative directly from the grid images, as proposed in $[16,17]$ for instance, reduces the number of parameters that govern the extracting procedure to one since only the size of the window used in the windowed Fourier transform used in this case drives the procedure. As a result, the link between the obtained strain field and the grid images is straightforward. This link has been characterized in [18] in the case of perfect grids. This enables one to tackle the problem of actual strain measurement in case of strong strain gradient with a suitable tool, namely deconvolution.

Deconvolution techniques are widely used in image processing but it seems that they have only seldom been employed in the field of mechanics [19, 20]. Concerning displacement and strain measurement, one of the main reasons is certainly that the "classic" procedure involves several non-linear steps and numerous parameters. Therefore, the retrieved mechanical quantities do probably not appear as the convolution of any kernel with the actual quantities. Knowing the kernel is however crucial to perform efficient deconvolution [21].

In this context, the aim of this paper is to show that deconvolution can be applied to enhance the ability of the grid method to detect and reliably quantify strain gradients. The paper is organized as follows. After a short recall on the grid method, the basics of deconvolution are described along with the characteristics of various deconvolution algorithms. The performance of these algorithms is then briefly reviewed and a numerical example is processed to study the limit and interest of deconvolution in the current context, especially when noise corrupts the grid images that are processed. Two short examples of actual strain map restoration picked in the recent literature are then shown to illustrate the procedure.

\section{Determining in-plane strain components from grid im- ages}

The objective here is to briefly recall the procedure used to determine in-plane strain components from grid images. In such images, the light intensity $s(x, y)$ at each point $(x, y)$ is a quasi-periodic signal characterized by two phases $\Phi_{x}$ and $\Phi_{y}$ which are defined along the $x$ and $y$ - directions, respectively. The variation of these phases between two images, denoted as $\Delta \Phi_{x}$ and $\Delta \Phi_{y}$, are related to the in-plane displacements $u_{x}$ and $u_{y}$ through the following equations [13]

$$
\left\{\begin{array}{l}
u_{x}=-\frac{p}{2 \pi} \times \Delta \Phi_{x} \\
u_{y}=-\frac{p}{2 \pi} \times \Delta \Phi_{y}
\end{array}\right.
$$

The in-plane strain components are therefore deduced from the phase derivatives variations 


$$
\left\{\begin{array}{l}
\epsilon_{x x}=-\frac{p}{2 \pi} \times \Delta \frac{\partial \Phi_{x}}{\partial x} \\
\epsilon_{y y}=-\frac{p}{2 \pi} \times \Delta \frac{\partial \Phi_{y}}{\partial y} \\
\epsilon_{x y}=-\frac{p}{2 \pi} \times\left(\Delta \frac{\partial \Phi_{x}}{\partial y}+\Delta \frac{\partial \Phi_{y}}{\partial x}\right)
\end{array}\right.
$$

Considering a given grid picture, the phases can be determined by calculating a windowed Fourier transform which writes as follows

$$
\begin{aligned}
\Psi(x, y, \theta) & =\int_{-\infty}^{+\infty} \int_{-\infty}^{+\infty} s(\xi, \eta) g_{\sigma}(x-\xi, y-\eta) e^{-2 i \pi f(\xi \cos (\theta)+\eta \sin (\theta))} \mathrm{d} \xi \mathrm{d} \eta \\
& =R(x, y, \theta)+i J(x, y, \theta)
\end{aligned}
$$

where $g_{\sigma}$ is a $2 \mathrm{D}$ window function of width $\sigma$. It is symmetric, positive, and integrates to 1 . In practice, this window can be a triangle, as suggested in [13], or a Gaussian, as in [17] to ensure differentiability. A Gaussian function is used in the current work. $\theta$ can be either equal to 0 or $\frac{\pi}{2}$, depending on the direction under consideration: $x$ or $y$, respectively. The actual phase variations $\Delta \Phi_{x}(x, y)$ and $\Delta \Phi_{y}(x, y)$ are approximated by calculating the following quantities denoted $\Delta \widetilde{\Phi_{x}}(x, y)$ and $\Delta \widetilde{\Phi_{y}}(x, y)$

$$
\left\{\begin{array}{l}
\Delta \widetilde{\Phi_{x}}(x, y)=\Delta \arctan \left(\frac{J(x, y, 0)}{R(x, y, 0)}\right) \\
\Delta \widetilde{\Phi_{y}}(x, y)=\Delta \arctan \left(\frac{J\left(x, y, \frac{\pi}{2}\right)}{R\left(x, y, \frac{\pi}{2}\right)}\right)
\end{array}\right.
$$

\subsection{The apparent phase and phase derivatives as the convolution of their actual counterparts}

An important result shown in [18] is the fact that each of the $\Delta \widetilde{\Phi_{i}}$ quantities, $i=x, y$, is nearly equal to the convolution of its actual counterpart $\Delta \Phi_{i}, i=x, y$, by the envelope $g_{\sigma}$ used in the windowed Fourier transform defined above. The same remark holds for the phase derivatives. Basically, this is true because displacements are small with respect to the pitch of the grid [18]. Thus

$$
\Delta \widetilde{\Phi_{i}} \simeq \Delta \Phi_{i} * g_{\sigma} \quad i=x, y
$$

with $\theta=0$ for $i=x$ and $\theta=\frac{\pi}{2}$ for $i=y$. In the same way

$$
\left\{\begin{array}{l}
\Delta \frac{\partial \widetilde{\Phi_{i}}}{\partial x} \simeq \Delta \frac{\partial \Phi_{i}}{\partial x} * g_{\sigma} \\
\Delta \frac{\partial \Phi_{i}}{\partial y} \simeq \Delta \frac{\partial \Phi_{i}}{\partial y} * g_{\sigma} \quad i=x, y
\end{array}\right.
$$

In terms of displacement and strain, plugging Eqs 5 and 6 in Eqs 1 and 2 leads to the displacement and strain components $\widetilde{u_{i}}$ and $\widetilde{\epsilon_{i j}}$ provided by the procedure, which can also be approximated by the convolution of their actual counterparts $u_{i}$ and $\epsilon_{i j}$ by $g_{\sigma}$. Thus 


$$
\widetilde{u_{i}} \simeq u_{i} * g_{\sigma}
$$

with $i=x, y$, and

$$
\widetilde{\epsilon_{i j}} \simeq \epsilon_{i j} * g_{\sigma}
$$

with $i j=x x, y y, x y$.

This property has two main consequences:

1. Any sudden variation of the actual displacement (resp. strain) distribution is smoothed by the phase (resp. phase derivative) extraction procedure. Hence resulting maps are automatically blurred in zones featuring high gradients. Consequently, the quantities provided by the grid image processing are lower than the actual ones over these zones;

2. Since such maps can be considered as maps of the actual quantity convolved by $g_{\sigma}$, one can restore them and find the actual quantity by deconvolution.

Remark 1 above mainly concerns strain components in practice, displacement gradients being very small within the framework of small deformations. Consequently, Remark 2 also mainly concerns strain components in practice. It must however be pointed out that it is only valid if no filtering (with polynomials for instance) is performed prior to strain calculation to remove noise. Indeed, the classic route for calculating strain components ([22] for instance) consists in:

1. extracting the phase and deducing the displacement field;

2. smoothing the displacement field with a suitable procedure to reduce noise;

3. differentiating the smoothed displacement field to deduce the strain field,

In this case, the number of parameters that govern the procedure to go from the grid images to the strain map increases. Moreover these parameters generally spatially change, so it is not possible to perform deconvolution. The idea here is to reduce this number as much as possible to be able to perform deconvolution and thus to retrieve the actual strain in case of strong strain gradients. For instance, Steps 1 and 2 above can be merged merely by adjusting the size of the Gaussian envelope used in the windowed Fourier transform. This is done by changing the value of $\sigma$. Even the three steps above can be merged by using explicit derivation, as in $[16,17]$ but the phase obtained at the end of Step 2 can also be numerically differentiated. In conclusion, only one main parameter may potentially govern the smoothness of the strain field obtained at the end of the procedure: $\sigma$. The larger the value of $\sigma$, the higher the smoothness of the strain field but the worst the spatial resolution in strain (defined here by the shortest distance between two points where independent strain measurements are obtained) and the stronger the weakening of the strain value in case of high strain gradients.

\subsection{Synthetic image}

A numerical example is discussed here to illustrate the fact that the difference between the phase derivative provided by the windowed Fourier transform and the actual one increases when the phase derivative gradient increases. The same example is then used throughout the paper to discuss the performance of various deconvolution techniques. 
It is assumed that the phase derivative $\frac{\partial \Phi_{y}}{\partial y}$ is a sine wave along the $y$-direction of the image, with a period slightly linearly changing along the $x$-axis. The idea under this assumption is to provide an image containing a rich (within certain limits) and regular frequency signature, but with a frequency nearly constant over the Gaussian kernel. The other phase $\Phi_{x}$ is assumed to be null. In practice, the period $p_{\text {wave }}$ of this sine wave is modelled by the following equation

$$
p_{\text {wave }}=p_{\text {wave }}^{\text {mini }}+\frac{p_{\text {wave }}^{\text {maxi }}-p_{\text {wave }}^{\text {mini }}}{L_{x}} \times x
$$

where $p_{\text {wave }}^{\min i}$ is the minimum value of the sine period and $p_{\text {wave }}^{\operatorname{maxi}}$ its maximum value. $L_{x}$ is the width of the image. Its height is denoted $L_{y}$ in the following. The phase amplitude is chosen in such a way that the amplitude of its derivative denoted $A_{\Phi^{\prime}}$ is constant. Thus

$$
\left\{\begin{array}{l}
\Phi_{x}=0 \\
\Phi_{y}=A_{\Phi^{\prime}} \frac{p_{\text {wave }}}{2 \pi} \sin \left(\frac{2 \pi}{p_{\text {wave }}}\left(y-\frac{L_{y}}{2}\right)\right)
\end{array}\right.
$$

and

$$
\left\{\begin{array}{l}
\frac{\partial \Phi_{x}}{\partial x}=\frac{\partial \Phi_{x}}{\partial y}=0 \\
\frac{\partial \Phi_{y}}{\partial x}=0, \frac{\partial \Phi_{y}}{\partial y}=A_{\Phi^{\prime}} \cos \left(\frac{2 \pi}{p_{\text {wave }}}\left(y-\frac{L_{y}}{2}\right)\right)
\end{array}\right.
$$

The interest of this phase derivative distribution is to see at a glance the response of various phase derivative calculation and restoration strategies. It is symmetric with respect to the horizontal midline. This feature will turn out to be useful in the following since the crosssection of the phase derivative map along this symmetry axis will be plotted to assess the performance of various deconvolution techniques. $p_{\text {wave }}^{\operatorname{mini}}$ and $p_{\text {wave }}^{\operatorname{maxi}}$ are chosen in such a way that the sine wave period gently decreases from the left to the right, so the period variation over the kernel remains negligible.

The phase and the derivative maps are shown in Figure 1 where $A_{\Phi^{\prime}}=1 E-03$ (which represents a typical value when strains are measured with this method), $L_{x}=4000$ pixels, $L_{y}=1000$ pixels, $p_{\text {wave }}^{\text {mini }}=10$ pixels and $p_{\text {wave }}^{\text {maxi }}=80$ pixels. The scale along the $x$ and $y$-axes is different to avoid too high an aspect ratio of the resulting image.

This phase is then used to build up a synthetic grid image for which the light intensity can be written as follows:

$$
s(x, y)=\frac{A}{2}\left(2+\sin ^{3}(2 \pi f x)+\sin ^{3}\left(2 \pi f y+\Phi_{y}(x, y)\right)\right)+n(x, y)
$$

where $n(x, y)$ is a Gaussian white noise of standard deviation $N$. Note that the signal is not encoded with a sine to illustrate that a pure sine is not rigorously necessary to correctly retrieve the phase derivative since only the first harmonic contains the sought information [13],[18]. The simulated grid image (not shown here) is built with $A(x, y)=A=2^{12}, 5$ pixels/grid period and quantization of the grey level to 12 bits. Such values are typical in real experiments carried out with a CCD camera. Note that the quantization step makes it impossible to perfectly retrieve the actual phase or phase derivative from the grid image. 


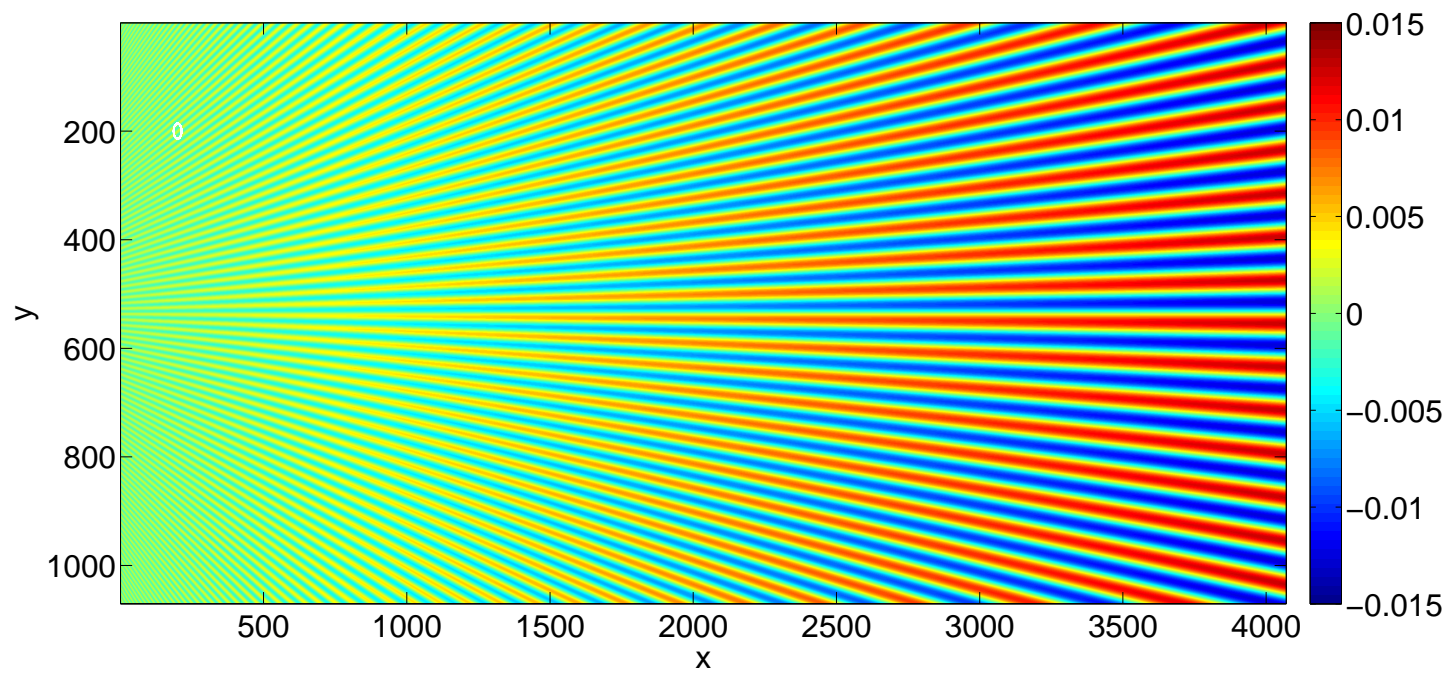

a- Phase $\Phi_{y}$

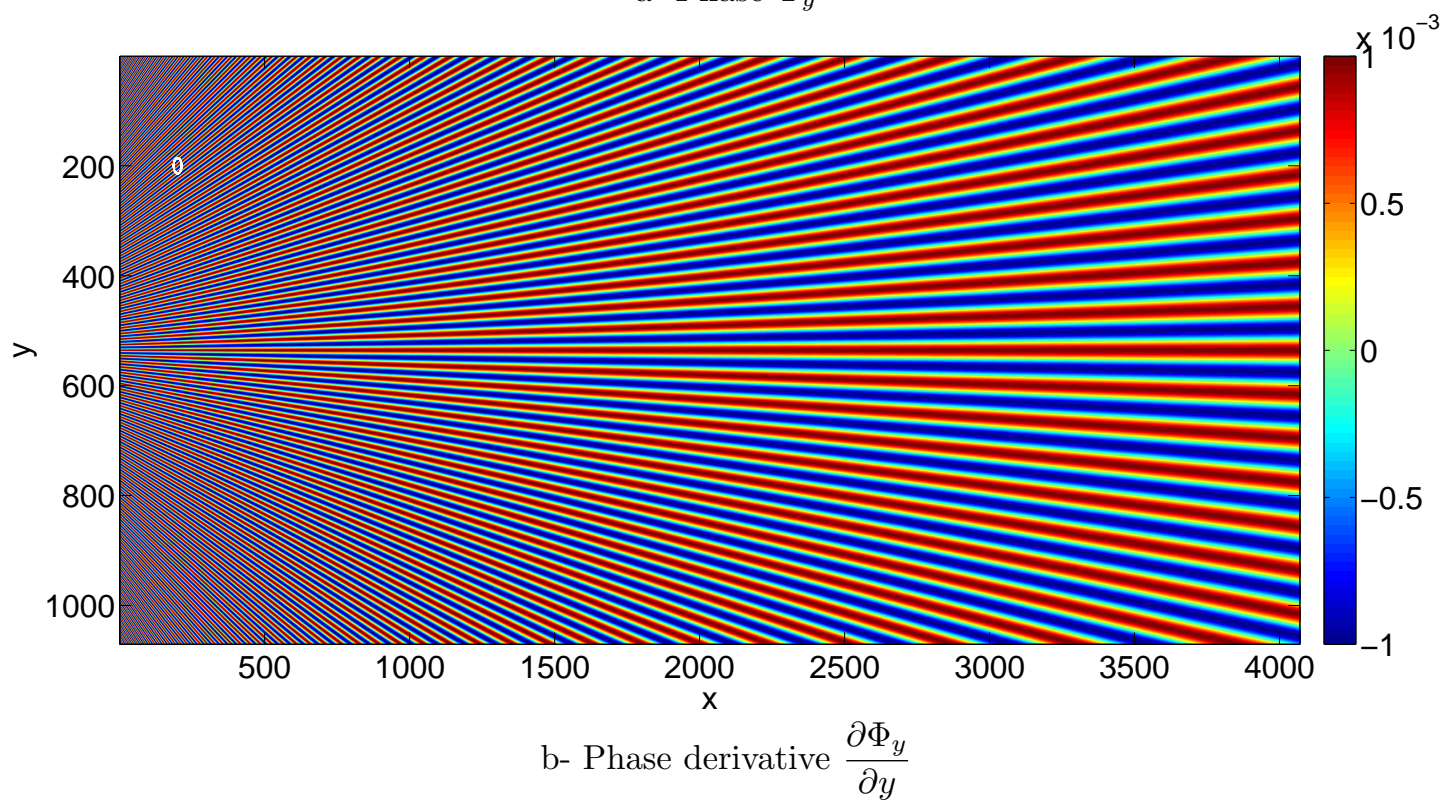

Figure 1: Reference phase derivative and phase maps 


\subsection{Convolution of the phase derivative}

The objective here is to illustrate and to quantify the fact that the phase derivative which is retrieved with the windowed Fourier transform is different from the actual one because of convolution. Parameter $\sigma$, which drives the window size, is equal to $\sigma=5$ pixels in these simulations, which is a typical value used in practice [17]. A top view of the envelope $g_{\sigma}$ is superimposed on the top left-hand side of the map shown in Figure 1-a and -b in order to more clearly compare the size of this envelope and the period of the waves. Since the scale along the $x$ and $y$ - axes is different, the circle turns into an ellipse in each map. The actual diameter of the circle is equal to $6 \times \sigma=30$ pixels, $\pm 3 \sigma$ being the usual width considered for a Gaussian envelope. It can be seen to the naked eye that the envelope covers several wave periods on the left-hand side (up to 3), thus correctly assessing the phase derivative is difficult.

The estimated phase derivative is shown in Figure 2-a. As may be easily seen since the amplitude of the reference phase derivative is constant, the estimated phase derivative is all the more impaired by the grid image processing that the frequency of the reference phase increases (see the left-hand side of the figure). The cross-section of the map along its symmetry axis (the horizontal midline defined by $y=500$ ) is plotted in Figure 2-b. The phase derivative should be constant along this mid-line (as the reference value) but it is clear that the estimated value is not, especially for high frequencies of the sine wave (left-hand side of the curve). The difference between estimated and reference phase derivative is finally shown in (Figure 2-c) to enable the reader to clearly assess this phenomenon. This simulation clearly illustrates the fact that deconvolution is necessary to try to restore as well as possible the phase derivatives to get reliable information from strain maps where significant strain gradients occur.

The phase derivative is all the more sensitive to this phenomenon that the width of the kernel envelope used for the windowed Fourier transform is significant compared to the period of the phase. This feature is illustrated in Figure 3-a where the cross-section of the phase derivative map along its symmetry axis $(y=500)$ is plotted for various values of $\sigma$. The higher the value of $\sigma$, the wider the Gaussian envelope and the worst the spatial resolution. In addition, the estimated phase derivative becomes more and more impaired by the procedure when $\sigma$ increases. The trade-off between resolution and spatial resolution is also illustrated in Figure 3-b where a noise has been added to the grid images used for plotting Figure 3-a. It is clear that the noise level observed on the phase derivative decreases when $\sigma$ increases, but the result becomes more and more impaired by the procedure at the same time.

Since the period of the phase derivative waves linearly decreases from the left to the right, the same results as above can be plotted with respect to a normalized period defined by the ratio $\xi$ between the period of the phase derivative waves and $\sigma$. The vertical lines correspond to boundary effects that can already be visible along the very left- and right-hand sides of Figure 3-a and -b. They are shifted toward the center of Figure 3-c because of the change in variable. They enable us to more clearly distinguish the domain over which they are defined. All the curves are perfectly superimposed but the number of normalized periods decreases when $\sigma$ increases, and therefore when noise decreases, thus illustrating that the size of the observed field is a third parameter which influences the quality of the result in addition to the spatial resolution and the resolution. The spatial resolution being defined here by the "width" of the Gaussian envelope $6 \sigma$, it can be seen that a loss of information slightly greater than $40 \%$ is obtained when the period of the waves is equal to this spatial resolution $(\xi=6)$ and nearly lower than $20 \%$ when this period is equal to twice this spatial resolution $(\xi=12)$. This highlights that a procedure able to restore the phase derivative would be very useful to get the actual value instead of quantities which are systematically impaired by the procedure employed to retrieve them. 

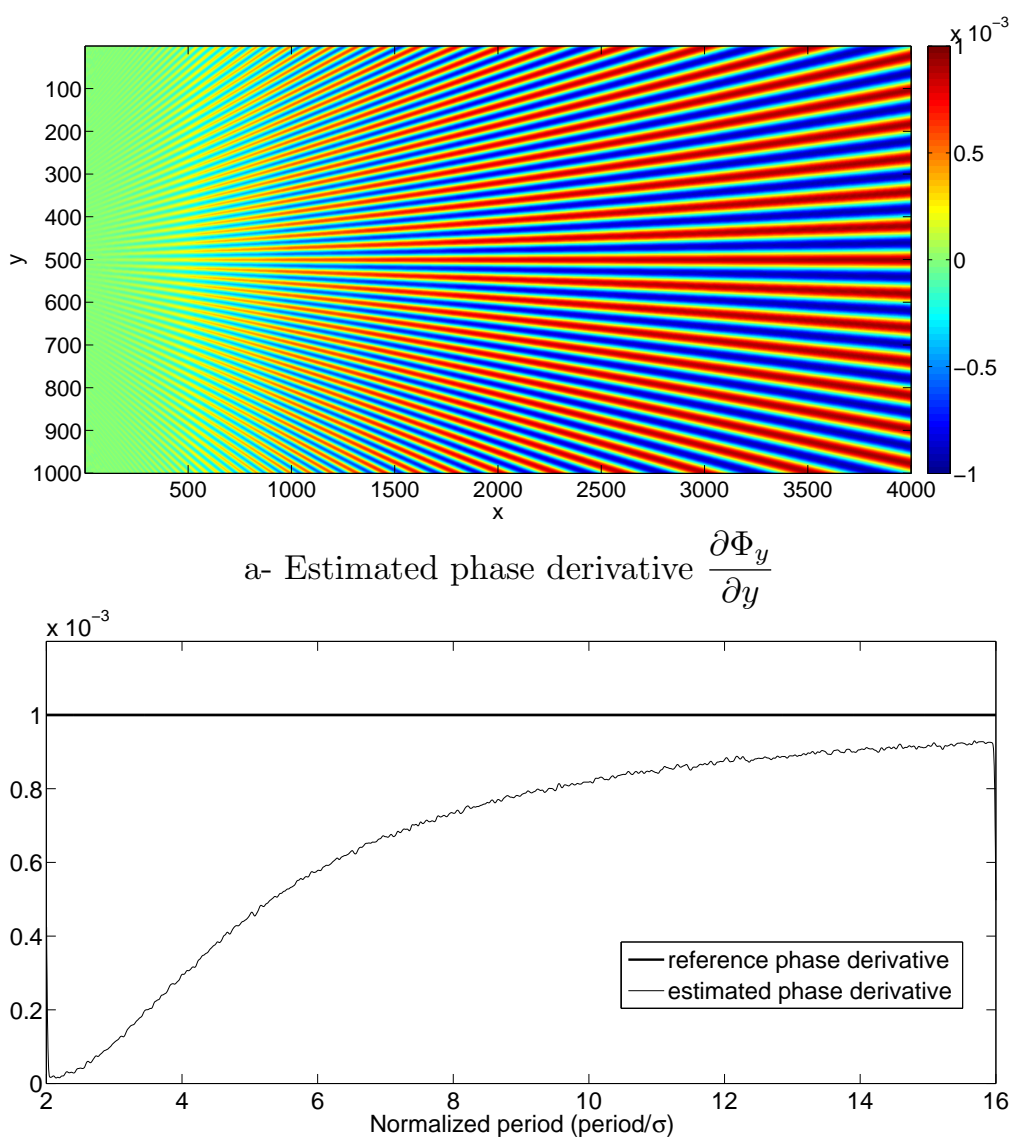

b- Cross-section of the estimated phase derivative map along the $x$-axis

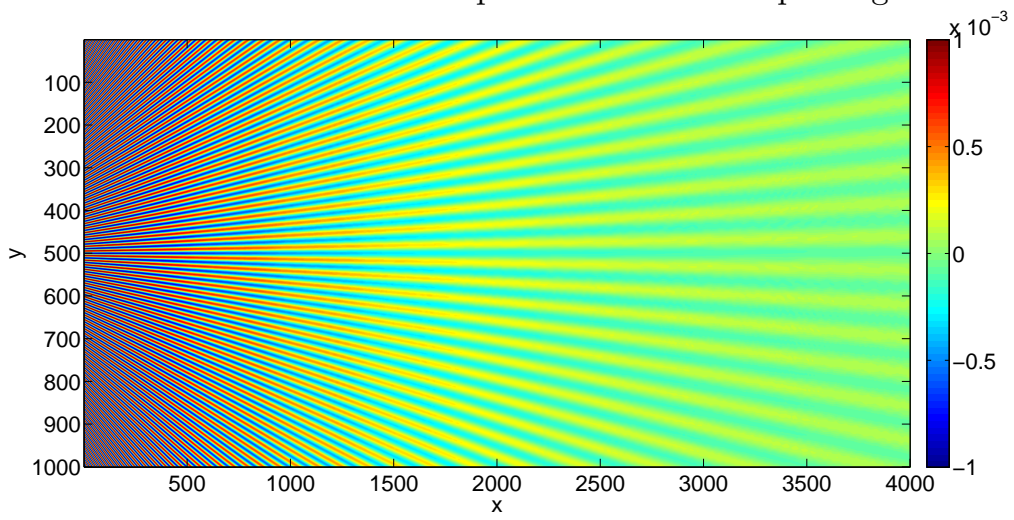

c- Difference between estimated and reference phase derivatives

Figure 2: Estimated phase derivative 


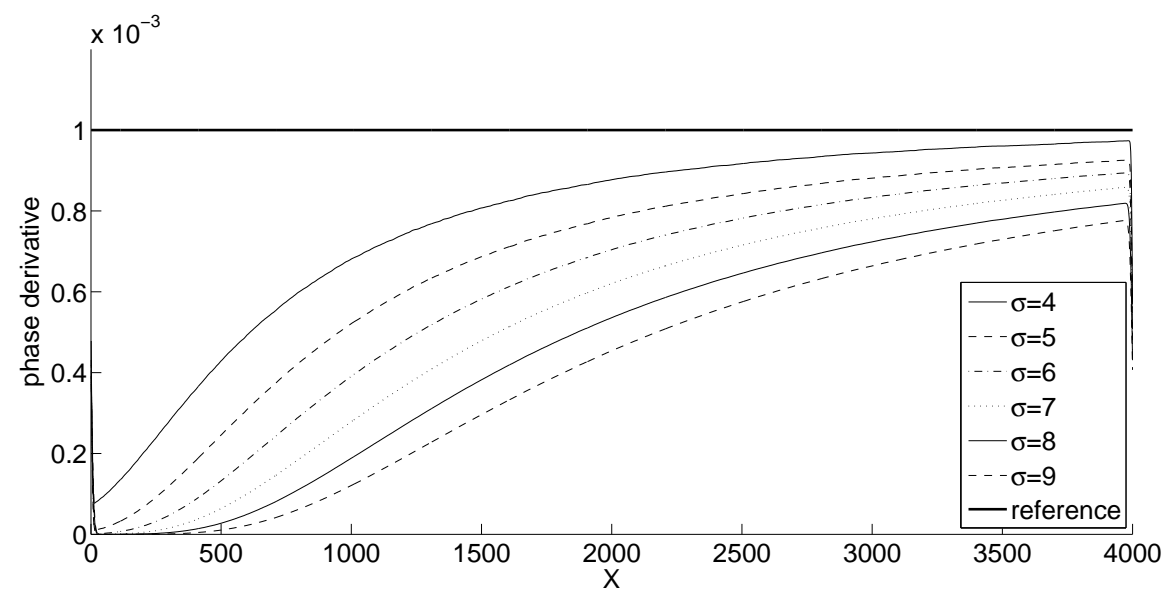

a- without noise $N=0$

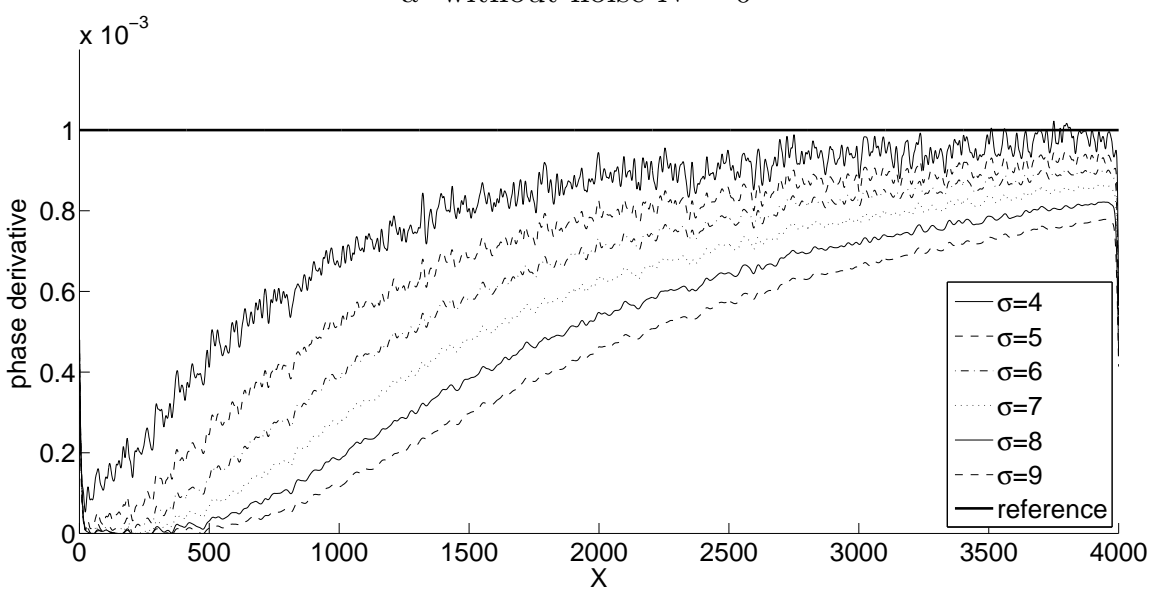

b- with noise $N=1$

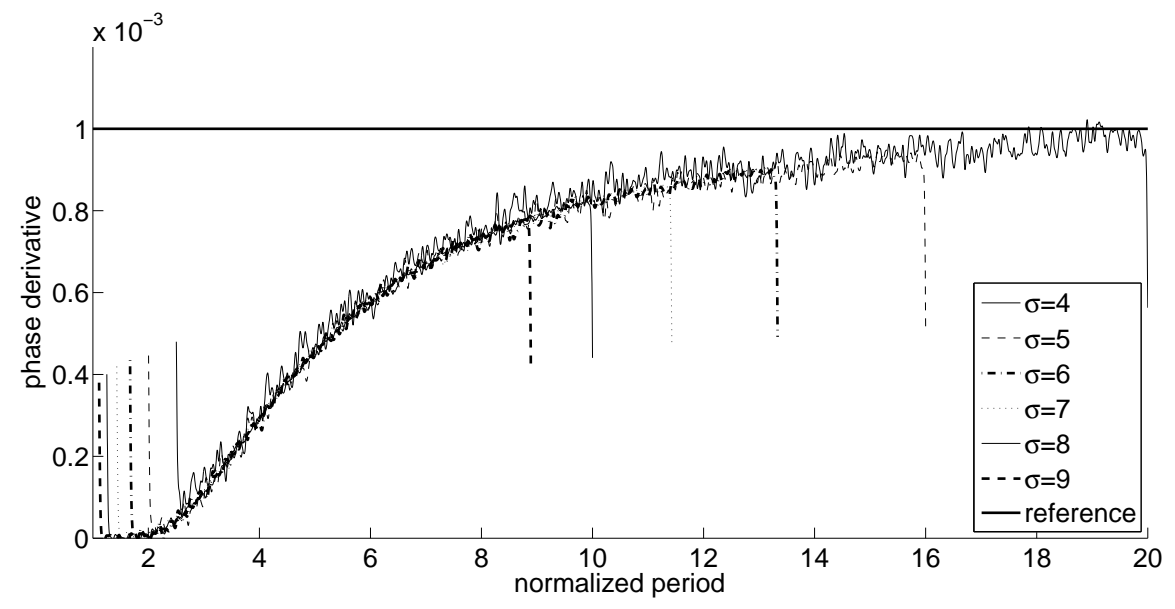

c- phase derivative vs. normalized period, without noise

Figure 3: Influence of $\sigma$ on the estimated phase derivative 


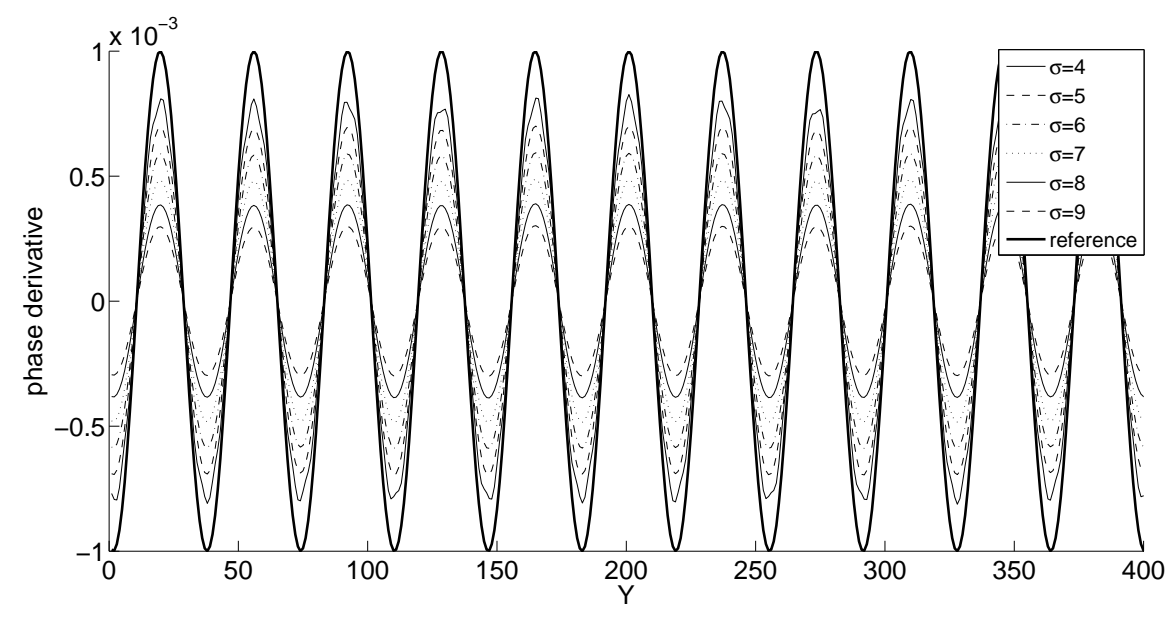

Figure 4: Cross-section of the phase derivative map at $x=1500$

The effect of convolution on the phase derivative is finally illustrated by a portion of the cross-section of the phase derivative map along the $y$ axis for $x=1500$. The result obtained for various values of $\sigma$ is shown in Figure 4. As may be seen, the difference between estimated and actual phase derivatives increases when $\sigma$ increases. Even for small values of $\sigma$, this difference is significant (about $20 \%$ of the reference value).

The objective is now to take advantage of the fact that $\frac{\partial \widetilde{\Phi_{y}}}{\partial y}$ can be considered as a convolution of $\frac{\partial \Phi_{y}}{\partial y}$ by $g_{\sigma}$ to retrieve $\frac{\partial \Phi_{y}}{\partial y}$ from $\frac{\partial \widetilde{\Phi_{y}}}{\partial y}$ by deconvolution. Various popular deconvolution techniques are briefly described in the following section. They are then applied to the example presented above and the obtained results are discussed. Some examples of strain maps picked in the recent literature serve finally as examples to briefly illustrate the interest but also the limits of deconvolution.

\section{Phase and phase derivative restoration by deconvolu- tion}

\subsection{Introduction}

The grid image is actually impaired by digital noise. Under the mild assumption that the image noise is a Gaussian white noise, it is shown in [18] that it yields a structured noise on the phase or its derivatives. In this section, the restoration problem is defined as follows

$$
u=u_{0} * g_{\sigma}+n
$$

where $u$ can be either the phase (or one of its derivatives) retrieved by the windowed Fourier transform, $u_{0}$ is the ideal phase (resp. one of its phase derivatives) and $n$ is the noise which corrupts the quantity to be deconvolved. $n$ is a spatially correlated Gaussian noise, which has been characterized for the phases and the phase derivatives in [18]. In this section, knowing $u$ and with some information about noise $n$, the aim of restoration is to estimate $u_{0}$. 
A large amount of literature is dedicated to deconvolution, either blind or non-blind. Let us name just the survey article [23] in the specific, yet representative field of astronomy. In the current case however, we are within the scope of non-blind deconvolution since the point spread function (PSF) which blurs the maps is well known a priori: this is merely the window envelope defined by $g_{\sigma}$. Even in this case which is easier to tackle than the case of blind deconvolution, it must be pointed out that non-blind deconvolution is an ill-posed inverse problem which is prone to noise amplification and artifacts such as the so-called ringing artifact [12]. Contrary to most of the studies in the field (although a recent survey paper [24] discusses the more limited denoising problem), the noise is not white in the problem of interest [18].

Four non-blind deconvolution techniques are briefly recalled here: direct deconvolution, regularized (Tikhonov) deconvolution [25], Richardson-Lucy deconvolution [26, 27] and Wiener filtering [28]. All these techniques have been used in turn to deconvolve the synthetic image presented and discussed above, the objective being to assess the efficiency of these techniques and their robustness even though the hypotheses under which some of them are developed are not fully satisfied.

\subsection{Brief presentation of the deconvolution techniques used}

In this section, $\widehat{f}$ denotes the Fourier transform of any function $f$. All the deconvolution methods described below have been tested in this study using standard Matlab functions for deconvolution from the Image Processing Toolbox [29], namely deconvreg, deconvwnr and deconvlucy. The arguments used in each case are briefly given for each deconvolution method.

\subsubsection{Direct deconvolution}

Neglecting the noise, $u=u_{0} * g_{\sigma}$ yields with Fourier transform:

$$
\widehat{u_{0}}(x, y)=\frac{\widehat{u}(x, y)}{\widehat{g_{\sigma}}(x, y)}
$$

Image $u_{0}$ is then retrieved by inverse Fourier transform. This method is called direct deconvolution. Since $g_{\sigma}$ is here a smooth function, $\widehat{g_{\sigma}}$ quickly vanishes for high frequencies. Dividing by $\widehat{g_{\sigma}}$ therefore accentuates high frequencies and possibly produces the ringing artifact along localized, "edge-like" features of the phase or its derivatives.

In the presence of noise, direct deconvolution yields $\widehat{u} / \widehat{g_{\sigma}}+\widehat{n} / \widehat{g_{\sigma}}$. This still amplifies high frequency noise artifacts in the retrieved image, which is even often overwhelmed by the noise. When no noise occurs, the Wiener filtering described below is equivalent to direct deconvolution. This latter technique was consequently implemented here using the Matlab function deconvwnr devoted to Wiener filtering, with the noise-to-signal ratio set to 0 .

\subsubsection{Regularized deconvolution}

A popular way to impose smoothness in deconvolution is to limit noise amplification by using Tikhonov regularization [25]. In this framework, the deconvolved image $u_{0}$ minimizes the following quantity

$$
\mathcal{F}(u)=\left\|u-u_{0} * g_{\sigma}\right\|_{2}^{2}+\lambda\left\|f\left(u_{0}\right)\right\|_{2}^{2}
$$

where $\left\|f\left(u_{0}\right)\right\|_{2}$ is a measure of "roughness" of $u_{0}$ and $\lambda$ controls the degree of roughness. In this paper, the popular Laplacian function $f\left(u_{0}\right)=\Delta u_{0}$ is used for this purpose. 
Note that if $\lambda=0$, since $\left\|u-g_{\sigma} * u_{0}\right\|_{2}^{2}=\left\|\widehat{u}-\widehat{u_{0}} \widehat{g_{\sigma}}\right\|_{2}^{2}$ (by Parseval theorem), minimizing $\mathcal{F}(u)$ yields $\widehat{u}=\widehat{u_{0}} \cdot \widehat{g_{\sigma}}$. In this case, the solution is therefore the same as in direct deconvolution. The Matlab function deconvreg with standard arguments was used here. In particular $\lambda$ was set automatically.

\subsubsection{Richardson-Lucy deconvolution}

Richardson-Lucy deconvolution $[26,27]$ is an iterative procedure defined by

$$
u_{i+1}(x, y)=u_{i}(x, y) \cdot \frac{u(x, y)}{u_{i} * g_{\sigma}(x, y)} * \overline{g_{\sigma}}
$$

where $\overline{g_{\sigma}}(x, y)=g_{\sigma}(-x,-y)$. Under Poisson noise assumption, $u_{i}$ converges to a maximum likelihood estimate of $u_{0}$. It is important that $u \geq 0$ to keep consistency with Poisson noise hypothesis.

In the current study case however, $u$ is not non-negative since we deal with phase and phase derivatives. Two possible workarounds are tested in this study:

1. The Richardson-Lucy deconvolution is used on $\exp (u)$ and the logarithm is then taken. This method is denoted LR 1;

2. The Richardson-Lucy deconvolution is used on $u-\min (u)$ and the result is normalized so that its mean is equal to the mean of the corrupted image $u$. This method is denoted LR 2.

Note however that in both methods, the noise on the non-negative image gray-values do not fit a Poisson distribution. This still proves to give satisfactory empirical results. Both versions were implemented with the Matlab function deconvlucy using standard arguments. The most critical one is the number of iterations whose influence is discussed in Section 3.5 below.

\subsubsection{Wiener filtering}

Wiener filtering [28] amounts to retrieve $u_{0}$ via

$$
\widehat{u_{0}}(x, y)=\frac{{\widehat{g_{\sigma}}}^{*}(x, y)}{\left|{\widehat{g_{\sigma}}}(x, y)\right|^{2}+M(x, y) / S(x, y)} \widehat{u}(x, y)
$$

where $z^{*}$ is the conjugate of the complex number $z$. $E$ denotes the expectation. $M(x, y)=$ $E\left(|\widehat{n}(x, y)|^{2}\right)$ is the mean power spectral density of the random noise $n$ and $S(x, y)=\left|\widehat{u_{0}}(x, y)\right|^{2}$ is the power spectral density of the (deterministic) ideal image $u_{0}$. Note that in the noisefree case, Wiener filtering boils down to direct deconvolution. Implementing Wiener filter necessitates to estimate both $M(x, y)$ and $S(x, y)$. Two approaches have been investigated here:

1. Assuming that $n$ is a white noise, then $M(x, y)=\operatorname{Var}(n)$. In [18], it is demonstrated that an accurate estimate of the variance of the noise on the phase maps $\phi_{x}$ and $\phi_{y}$ is:

$$
\operatorname{Var}(n)=\frac{N^{2} \Delta x \Delta y}{8 \pi \sigma^{2} K^{2}}
$$

where $N^{2}$ is the variance of the Gaussian white noise impairing the grid image. $K=$ $\left|d_{1}\right| A / 2$, where $d_{1}$ is the first Fourier coefficient of the $\sin ^{3}$ function, and $A$ is as in 
Eq. (12). $\Delta x \Delta y$ is the area of one pixel. It is equal to one here since all the dimensions are given in pixels, so it vanishes in this expression.

It is also shown that the variance of the noise on the phase derivative maps in the $x-$ and $y$ - directions is:

$$
\operatorname{Var}(n)=\frac{N^{2} \Delta x \Delta y}{16 \pi \sigma^{4} K^{2}}
$$

Besides, $S(x, y)$ is approximated by $|\widehat{u}(x, y)|^{2}$. This method is denoted hereafter WF 1 .

2. The autocorrelation function $\rho$ of the noise on $u$ is assumed to be given a priori, using the results presented in [18]. The well-known Wiener-Khinchin theorem yields $M(x, y)=$ $\widehat{\rho}(x, y)$. Concerning $S$, it is still approximated by $|\widehat{u}|^{2}$. This method is denoted WF 2 . hereafter.

Both Wiener filter variants were implemented with the Matlab function deconvwnr, each time with arguments suited to the corresponding variant.

\subsection{Deconvolution of the phase derivative of the synthetic example. Comparison between various techniques}

The objective here is to apply deconvolution to restore the phase derivative map discussed in Section 2.2 using the methods presented in Section 3.2. The efficiency of these methods is assessed by calculating the difference between deconvolved and reference phase derivatives, first assuming that no noise corrupts the grid images, and then with an additional noise to observe robustness. These so-called error maps, obtained by subtracting the phase derivative maps and the reference map, are used to assess the obtained result. $\sigma=5$ pixels is chosen in all these simulations to keep the spatial resolution constant. The Richardson-Lucy technique being iterative, $n_{i t}=10$ iterations are taken in all cases for these first attempts but the influence of this parameter is discussed in more details in Section 3.5 below.

Results obtained without noise added to the grid image are gathered in Figure 5. The Wiener filter boiling down to direct deconvolution when no noise is present, it is not employed here. Comparing the four error maps with that shown in Figure 2-c shows that a wide portion of the phase derivative map is restored. Some significant edge effects are clearly visible in the direct case and, to a lesser extend, in the regularized deconvolution case. Boundary effects along the vertical border occur only in the first case. Remarkably, the regularized deconvolution provides a map of nearly null error (to the naked eye) over a wider portion of the image compared to the other techniques. Hence even small details that take place on the left-hand side of the phase derivative map are restored in this case. For the three other techniques, a zone roughly defined by $x<500$ is not correctly restored. Beyond this threshold value, the error is nearly null in all cases. The fringe pattern is however slightly recognizable in the RL 2 error map.

The performance of the different methods can be compared by calculating the peak signal to noise ratio $(P S N R)$ over a certain zone defined by the white rectangle shown in Figure 5-d. The $P S N R$ value is calculated over this rectangle throughout this paper. This quantity is equal to $P S N R=20 \log _{10} \frac{D}{R M S E}$, where $R M S E$ represents the root mean square error calculated over this zone and $D$ is the dynamics of the grid image. The zone is defined away from the edge of the map to avoid boundary effects. The $P S N R$ value reflects here the quality of deconvolution in a zone where the error is stabilized with respect to the effect of the sine wave frequency on the deconvolved map. The PSNR values obtained with the four deconvolution techniques are represented in Figure 6-a along with their counterpart calculated with the image before 


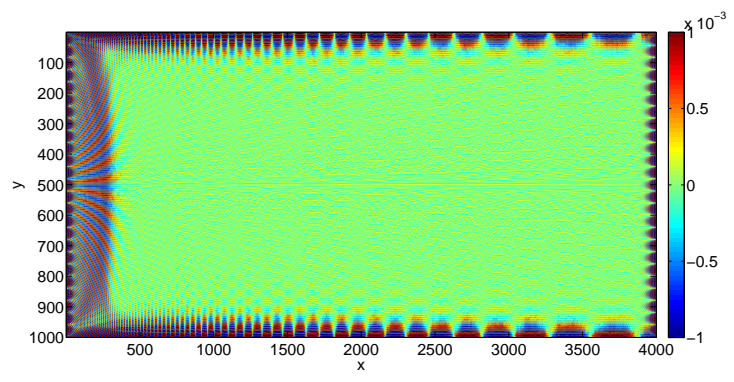

a- Direct deconvolution

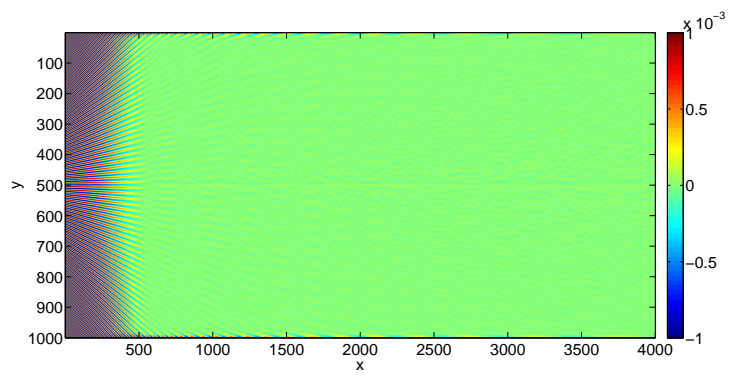

c- Richardson-Lucy, logarithmic (RL 1)

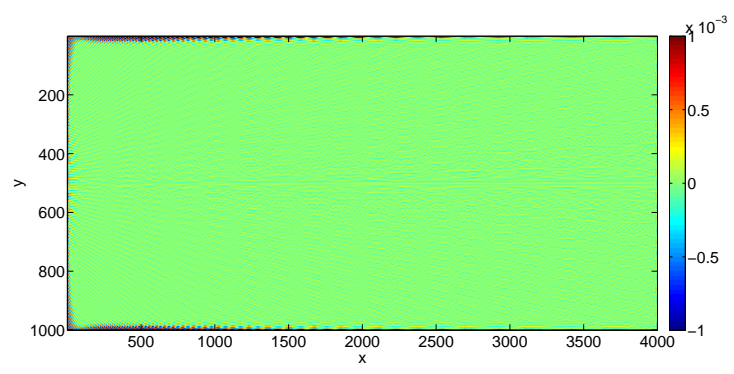

b- Regularized deconvolution

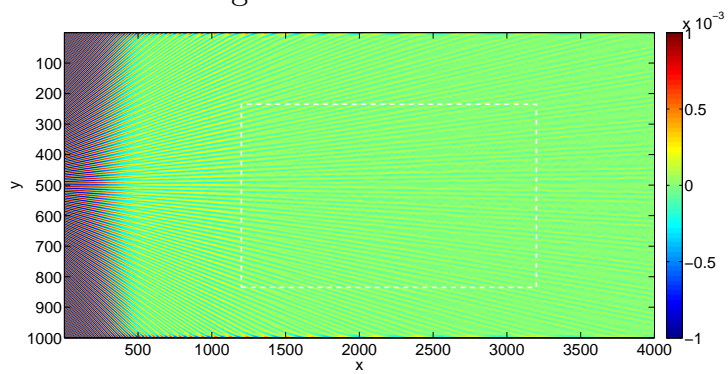

d- Richardson-Lucy, linear (RL 2)

Figure 5: Difference between deconvolved and reference phase derivatives obtained with various deconvolution strategies. Noise amplitude $=0$

deconvolution (see Figure 2-c) for comparison purposes (first bar on the left-hand side of the figure). As may be seen, all techniques lead to an improvement of the $P S N R$ value, but the best results are obtained with RL 1 .

The influence of the frequency of the phase derivative on the quality of the results can be assessed by examining the cross-section of the previous figures along the horizontal symmetry axis, where the reference phase derivative is constant whatever the sine wave frequency. The obtained result is shown in Figure 6-b where the result given by all the methods are superimposed. The boundary effects along the vertical border are clearly visible with direct deconvolution. It is also worthy noting that this technique provides a noisy distribution away from the borders. This is consistent with the conclusion drawn from the PSNR maps. On the left-hand side, the RL 1 and RL 2 curves sharply increase at a fairly linear rate which is nearly the same for both variants. In addition, RL 1 then significantly overestimates the phase derivative (between $x=500$ and $x=1200)$. In all cases however it is worth mentioning that the actual value is reached before $x=500$. At that point, it can be checked with Eq. 9 that the period of the sine wave $p_{\text {wave }}$ is slightly lower than $2 \sigma$, which is equal to one-third of the spatial resolution since the latter is assumed to be equal to $6 \sigma$. This result is very interesting since convolution induces a bias of about $20 \%$ in the phase derivative for a sine wave period equal to twice the spatial resolution, as discussed in Section 2.3 above.

The robustness of these different deconvolution strategies to the noise in the grid image is a key-issue. It has been examined by processing in turn the grid image corrupted by various noise levels. Figures 7-8 show a set of maps obtained with the same procedures as in Figure 5 . The only difference is the fact that a noise (zero mean, amplitude $N=3$ and $N=5$ for Figure 7 and 8 , respectively) has been added to the grid image modelled by Eq. 12. It clearly appears that the strategies described above exhibit a different response when the grid image is corrupted by 

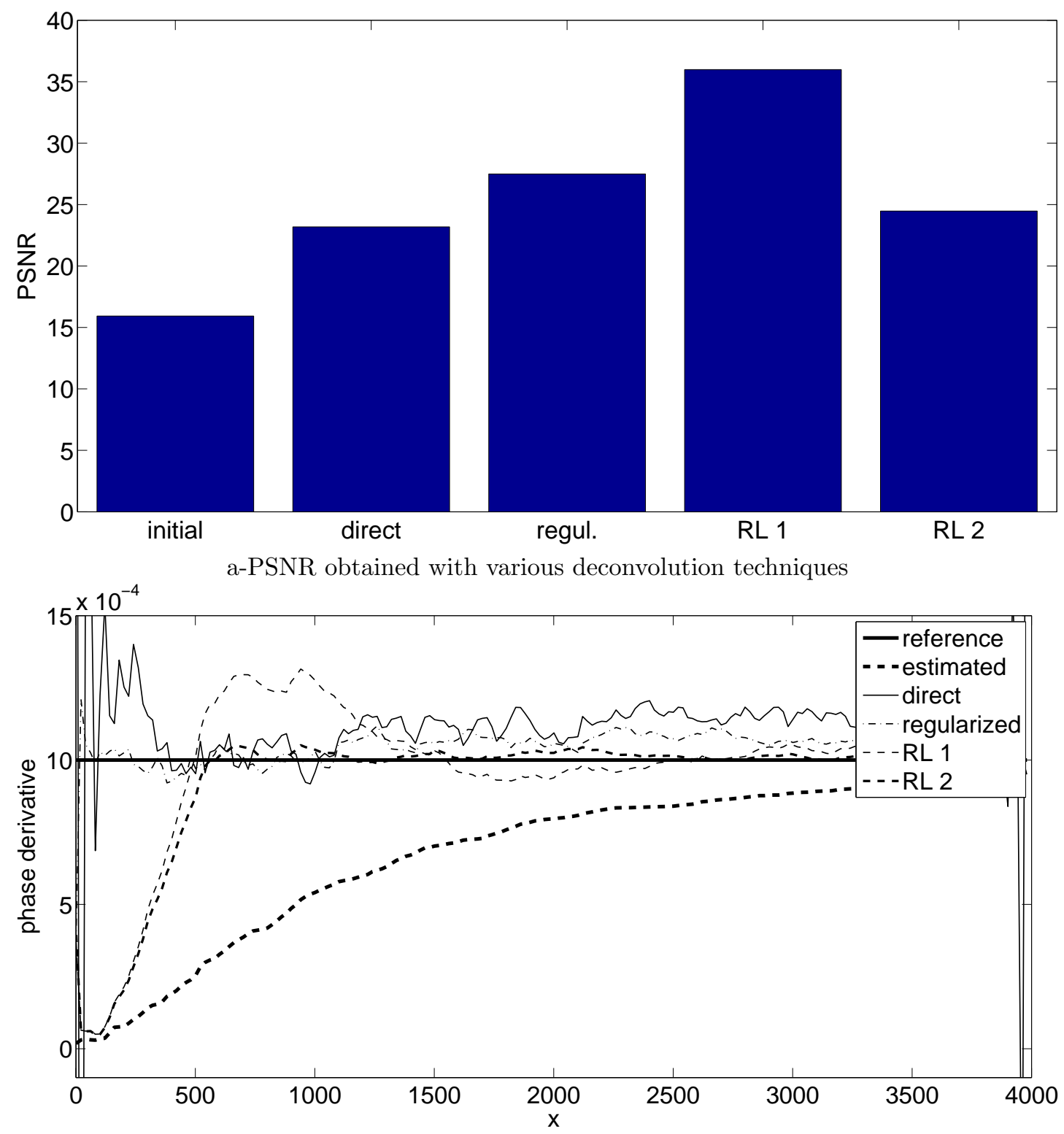

b- cross section of the error maps along the horizontal symmetry axis

Figure 6: Comparison of the results obtained with various deconvolution techniques. No noise 


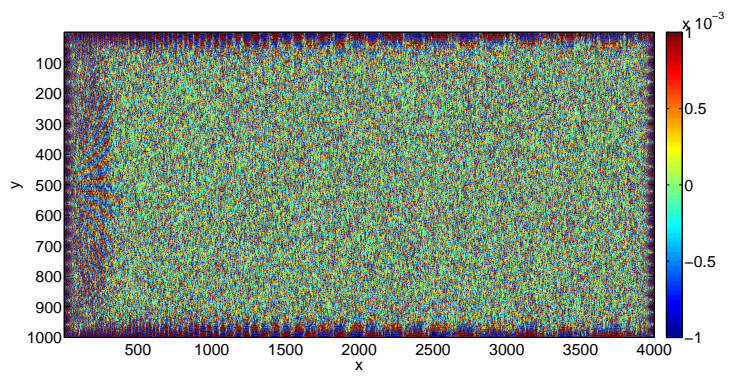

a- Direct deconvolution

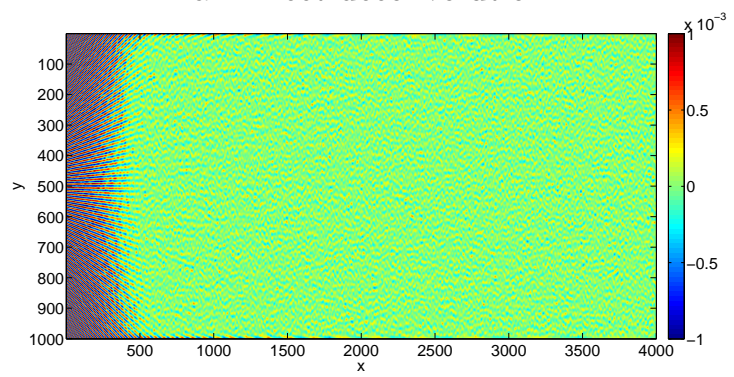

c- Richardson-Lucy, logarithmic (RL 1)

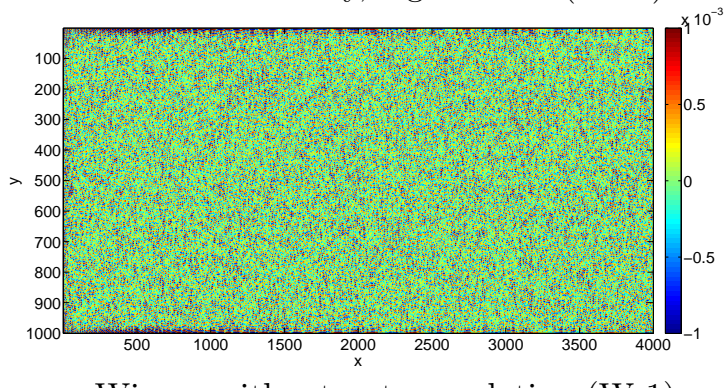

e- Wiener without autocorrelation (W 1)

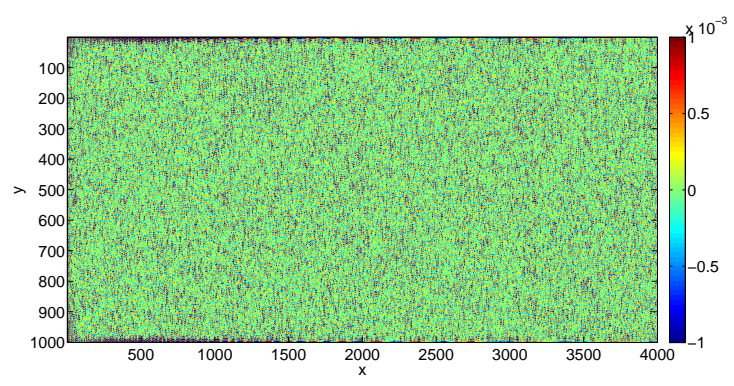

b- Regularized deconvolution

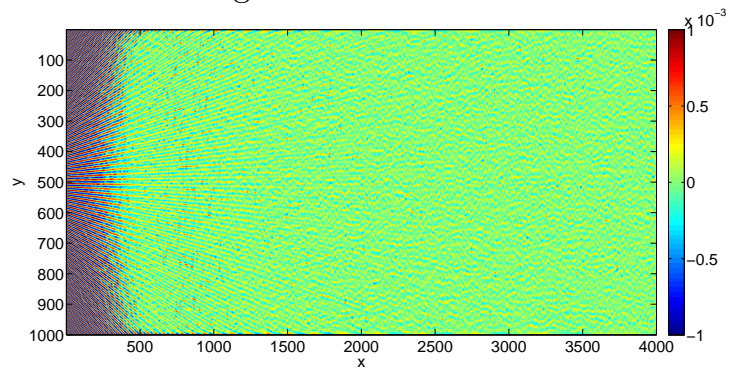

d- Richardson-Lucy, linear (RL 2)

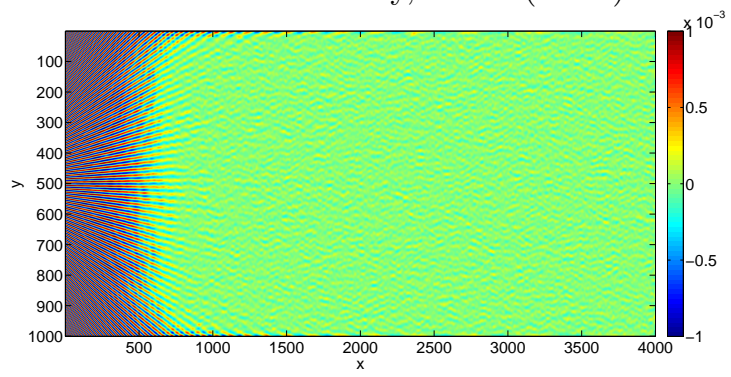

f- Wiener with autocorrelation (W 2)

Figure 7: Difference between deconvolved and reference phase derivatives obtained with various deconvolution strategies. Noise amplitude $=3$

noise. Figures 7-a,-b,-e and Figures 8-a,-b and -e clearly show that the direct, regularized and W 1 deconvolution strategies are not robust whereas the other ones (RL 1, RL 2 and W 2) still provide acceptable results. The last one (W 2) gives the less noisy phase derivative map, but details featuring a high frequency are not so well restored compared to the two techniques based on the Richardson-Lucy algorithm.

The performance of the techniques in each case can also be assessed with the $P S N R$ value. This quantity is represented in Figure 9. W 1 and W 2 appear only in case of noise, as justified above. The obtained results confirm the trends observed to the naked eye in Figures 7-8:

- The PSNR value decreases when the noise amplitude increases. This is quite logical;

- RL 1 provides the best results when no noise occurs;

- W 2 outperforms the other techniques when noise corrupts the grid images.

The latter result is obtained in a case for which noise has been correctly characterized to 


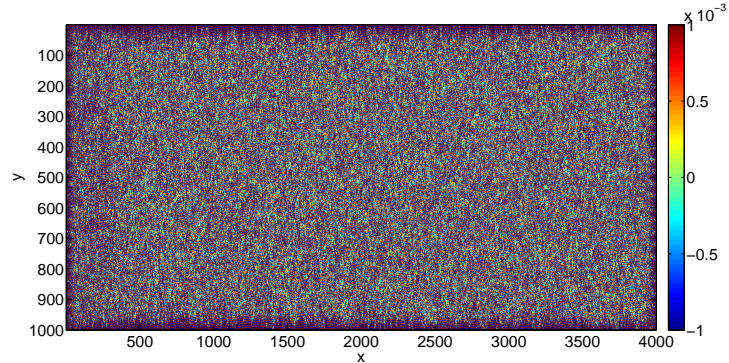

a- Direct deconvolution

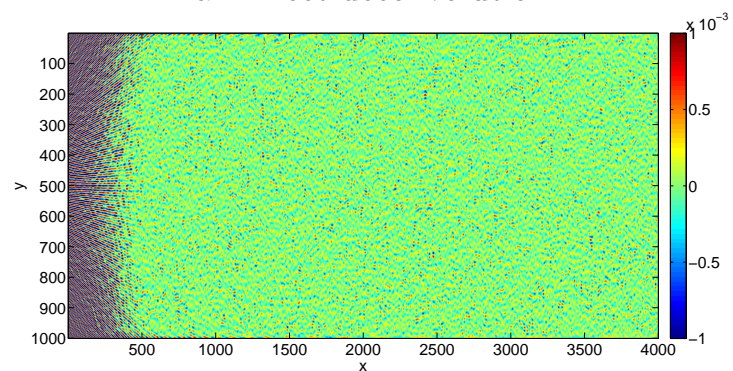

c- Richardson-Lucy, logarithmic (RL 1)

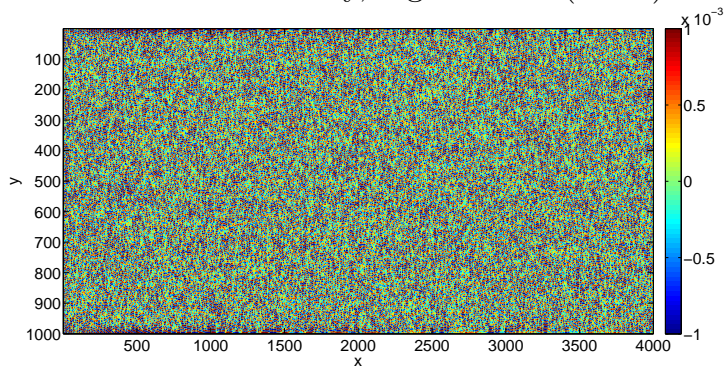

- Wiener without autocorrelation (W 1)

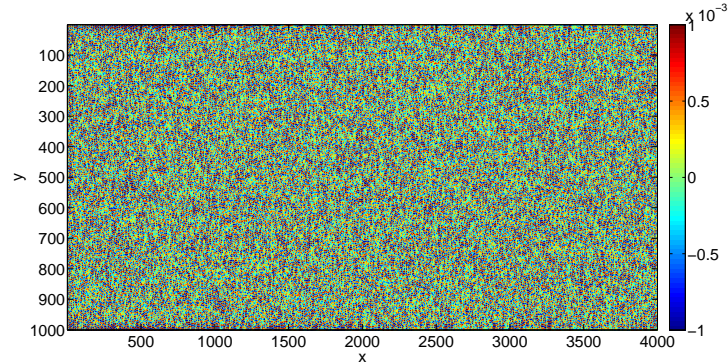

b- Regularized deconvolution

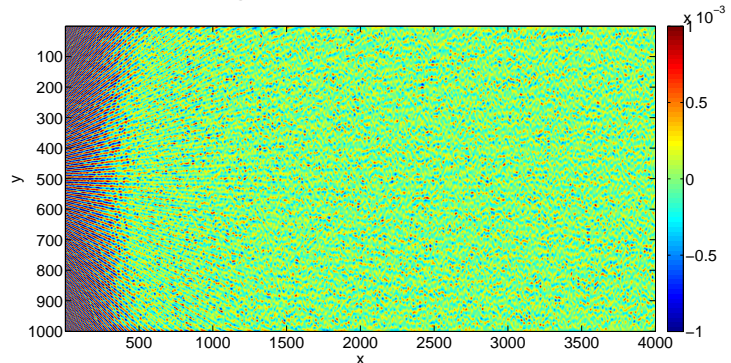

d- Richardson-Lucy, linear (RL 2)

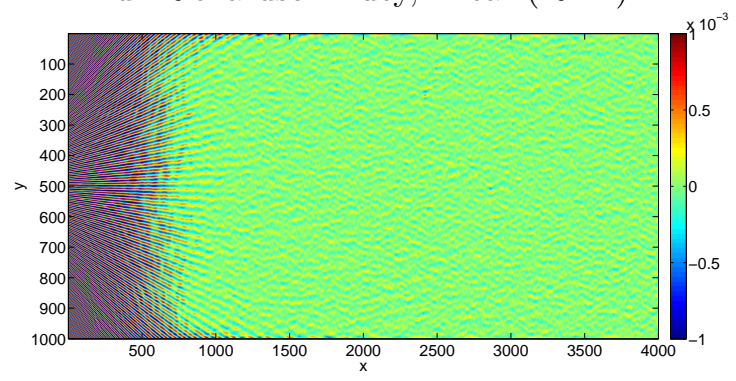

f- Wiener with autocorrelation (W 2)

Figure 8: Difference between deconvolved and reference phase derivatives obtained with various deconvolution strategies. Noise amplitude $=5$ 


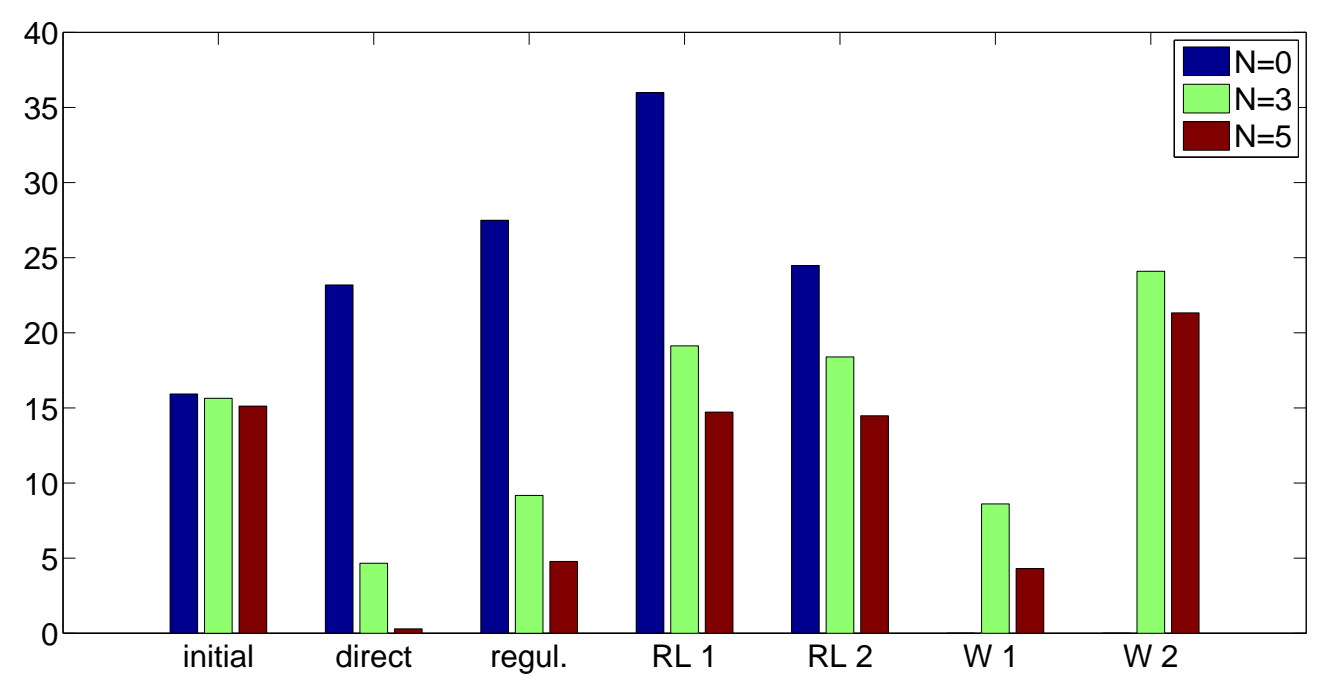

Figure 9: PSNR for various deconvolution techniques and noise levels

feed the filter. Indeed WL 1, which also relies on the Wiener filter, provides poor results because noise is not correctly characterized in this case (autocorrelation is not taken into account). This means that, in practice, the Wiener filter should be used with caution if noise is not correctly assessed. RL 1 or RL 2 should be used instead in this case since they still give acceptable results.

\subsection{Optimal choice of $\sigma$ for $\mathrm{W} 2$}

The previous results are obtained with a given value of $\sigma: \sigma=5$ pixels, but one can easily guess that this parameter also influences the results. Indeed, increasing $\sigma$ reduces noise level and therefore facilitates deconvolution. However this also reduces the amplitude of the convolved phase derivative level and it is no wonder that retrieving the actual one becomes more difficult if the value of $\sigma$ becomes too large. In addition, this also contributes some blobs to appear in the phase derivative map because the larger the $\sigma$, the larger the range of noise correlation, as discussed in [18]. These "blobs" may then be mistaken for fictitious local events that are then restored by deconvolution and this should obviously be avoided as far as possible. As a conclusion, choosing at best the value for $\sigma$ is a trade-off between various constraints. This issue has been investigated here by examining the influence of $N$ and $\sigma$ the $P S N R$ value.

Figure 10 shows the $P S N R$ value for various noise levels and values of $\sigma$. These curves are plotted for W2 and for the initial phase derivative maps. Six noise levels are considered in each case. As may be seen, the curves obtained with W 2 regularly decrease when the noise level increases, as expected. More interestingly, there is an optimal value lying between $\sigma=7$ pixels and $\sigma=9$ pixels, depending on the noise level. Considering that $\sigma=8$ pixels is optimal whatever the noise seems to be a sound choice for the following calculations.

The curves obtained without deconvolution decrease when $\sigma$ increases. This is quite logical since it means that convolution smoothes the resulting phase derivative map, thus increasing the error made when the size of the kernel increases because details are obtained with a lower quality. It is also worth mentioning that the effect of noise is hidden behind this phenomenon because the error due to smoothing is much greater that than that due to noise. Finally, it is 


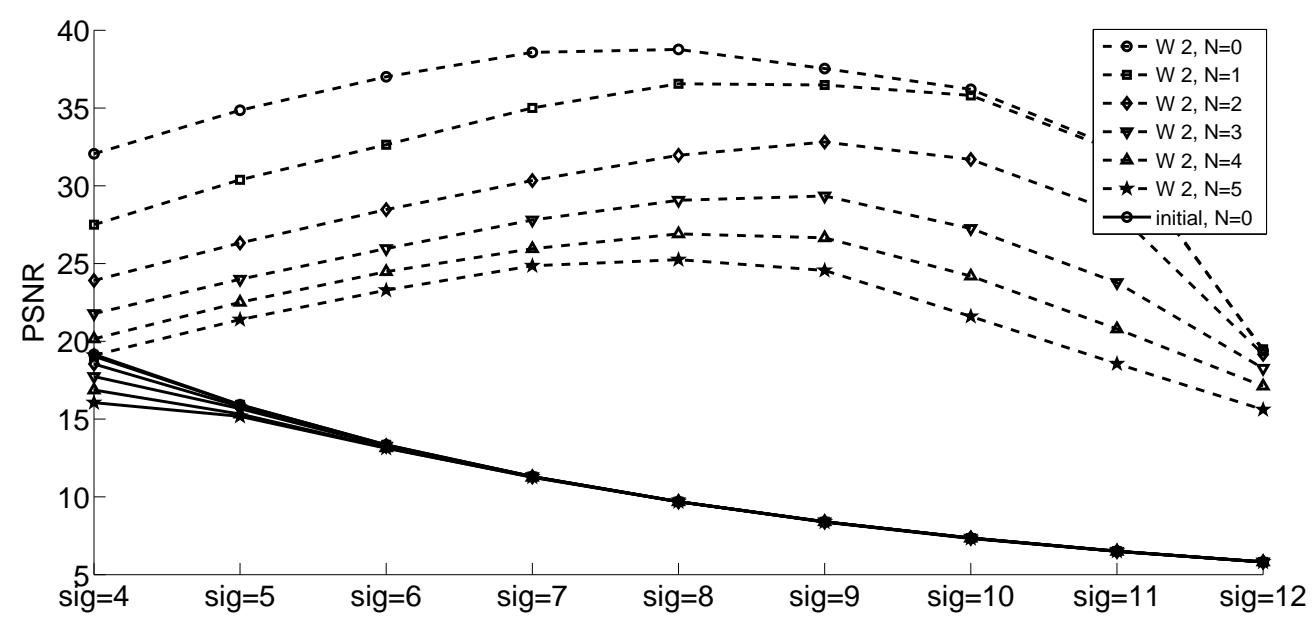

Figure 10: PSNR for $\mathrm{W} 2$, various values of $\sigma$ and noise levels

also clear that the $P S N R$ value is greater for $\mathrm{W} 2$ than for the initial image whatever the noise level, so it means that deconvolution with $\mathrm{W} 2$ remains stable in this case.

\subsection{Optimal choice of the number of iterations for RL 1}

The Richardson-Lucy algorithm being iterative, the number of iterations $n_{i t}$ must be chosen. For the same reason as above $\sigma$ must also be chosen. Since this quantity directly influences the noise level in the phase derivative map, it must be adjusted to limit this effect whilst limiting blobs due to correlated noise, as explained above. This issue has been investigated by calculating the PNSR value for various values of $\sigma$ and $n_{i t}$, the noise amplitude $N$ being given. Three cases have been investigated herein: $N=0,3,5$. The obtained results are shown in Figure 11. Comparing the PSNR values in the three cases confirms that noise significantly impairs the results, but it is worth mentioning that the maximum $P S N R$ value for the highest noise level (nearly $23.92 \mathrm{~dB}$ for $\sigma=10$ pixels, $n_{i t}=20$ ) remains much higher than that obtained with the raw phase derivative map (7.34 dB, see first column in Figure 11-c, $\sigma=10$ pixels). This shows that deconvolution still dramatically improves the phase derivative map despite noise. Another shrinking fact is that the optimal choice for the $\sigma$ and $n_{i t}$ parameters corresponds to a series of points located along the ridge of a 3D surface. This ridge slightly moves downwards left as the noise level increases. If the noise level remains unknown in practice, it is possible to choose a "reasonable value" for $\sigma$ from these figures: $\sigma=8$ pixels, and then launch the deconvolution programme with $n_{i t}=10$.

The fact that a maximum value for $P S N R$ exists for a given value of $\sigma$ means that beyond this maximum, the procedure restores some details in the phase derivative map which are sequels of the noise correlated by image processing and not zones of actual phase derivative concentration. This feature is illustrated in Figure 12 where the error map over the white rectangle shown in Figure 5 -d is plotted. The benefit of deconvolution is clearly visible since the residual fringes (difference between estimated and reference phase derivative) have disappeared, details in the phase derivative map being now restored, but noise amplitude also increases when $n_{i t}$ increases. This is quantified by the $P S N R$ value (reported in the caption of each sub-figure) which is greater for $n_{i t}=500$ iterations than that obtained before deconvolution: details are 


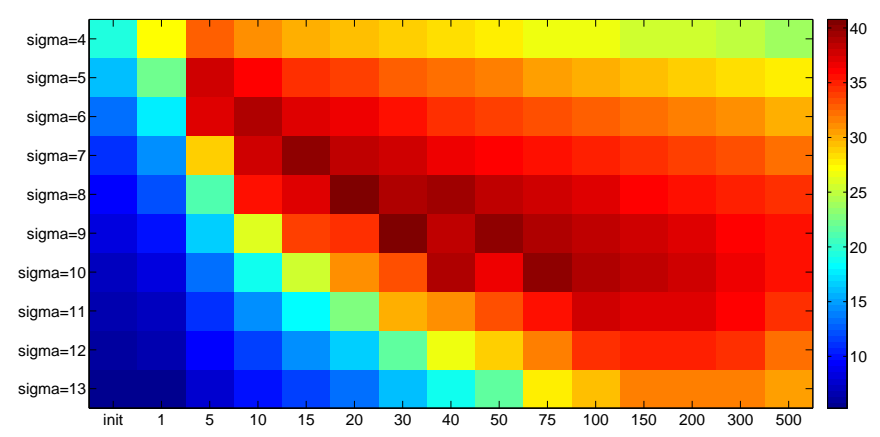

a- $N=0$
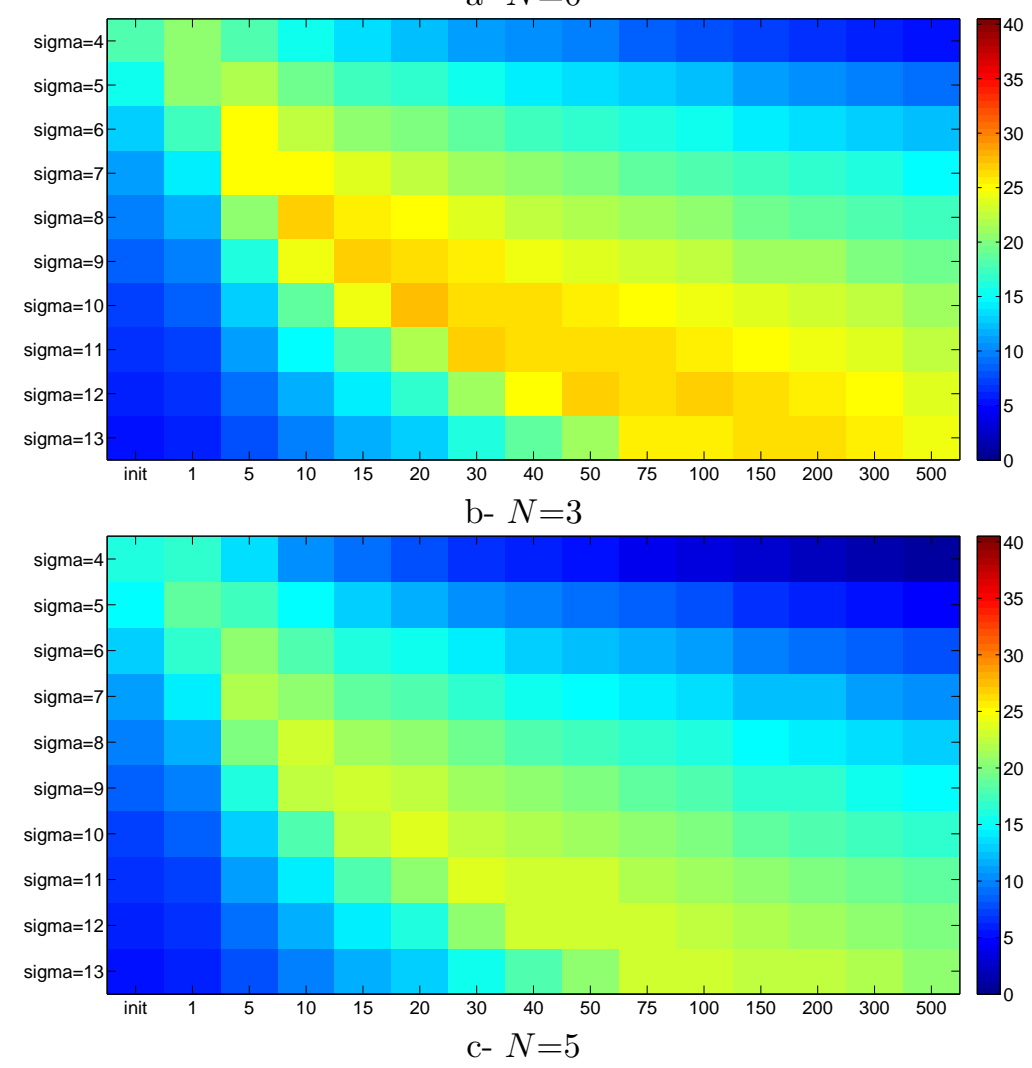

Figure 11: PSNR for RL 1, various values of $\sigma$ and $n_{i t}$ 
restored but noise also increases for large values of $n_{i t}$.

\subsection{Conclusion}

Some examples of restored phase derivative maps are shown in Figure 13 to conclude this section. The estimated phase derivative map is shown in Figure 13-a. One can see that the information progressively vanishes when moving from the right to the left, which illustrates the negative impact of convolution when the frequency of the details increases. The effect of noise on the grid image can be assessed in Figure 13-b. The two figures above serve then as input images for the deconvolution techniques. Applying RL 1 in the noiseless case leads to the map in Figure 13-c. Compared to the map located just above, it can be seen that a wide portion of the image is restored. A thin band located at the very left remains inaccessible even after deconvolution. The influence of noise can be assessed in Figure 13-d when W 2 (the best technique in this case after the results obtained above) has been employed, but with $\sigma=5$ pixels which is not the optimized value. Again, details are restored but noise clearly corrupts the resulting map. This result is improved by increasing $\sigma$, as suggested by the conclusion of the simulations in Sections 3.4 and 3.5 above. RL 1 provides less noisy results than W 2, but it can be checked that the error is greater for RL 1 . In addition, the zone which remains unrestored is greater for RL 1 than for W 2. 


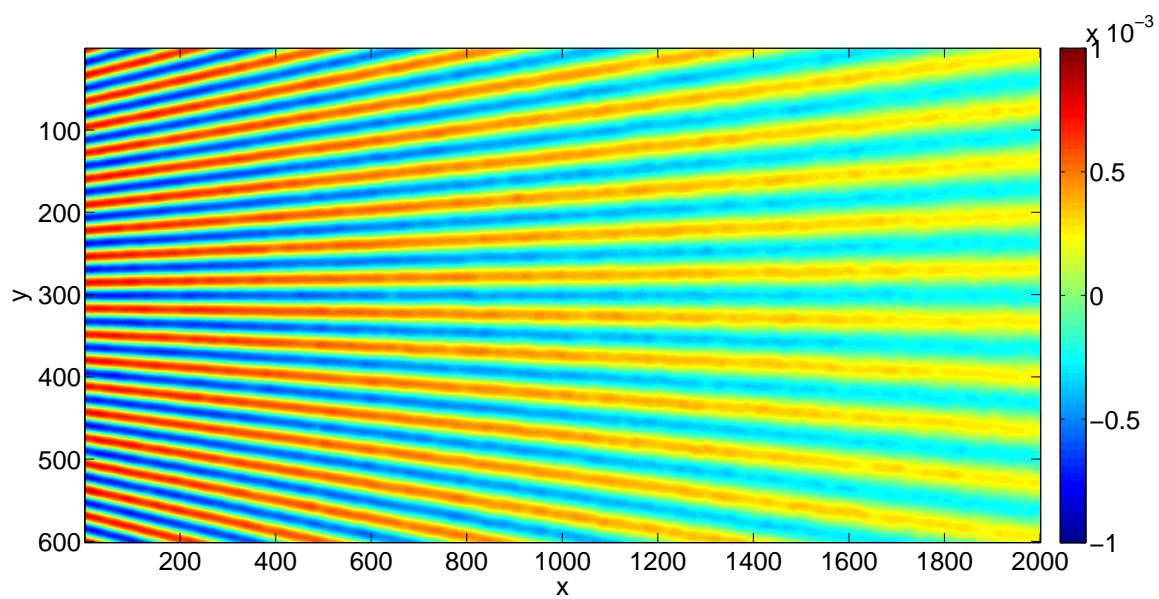

a- difference between estimated and reference phase derivative maps. PSNR $=9.68 \mathrm{~dB}$

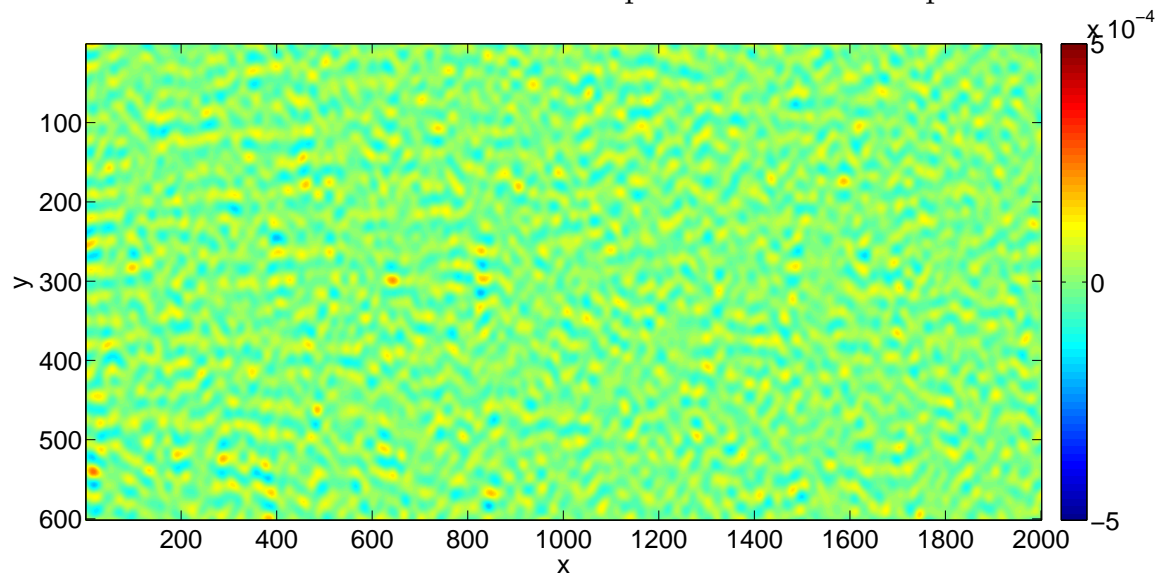

b- difference between deconvolved and reference phase derivative maps. RL $1, n_{i t}=10$ iterations.

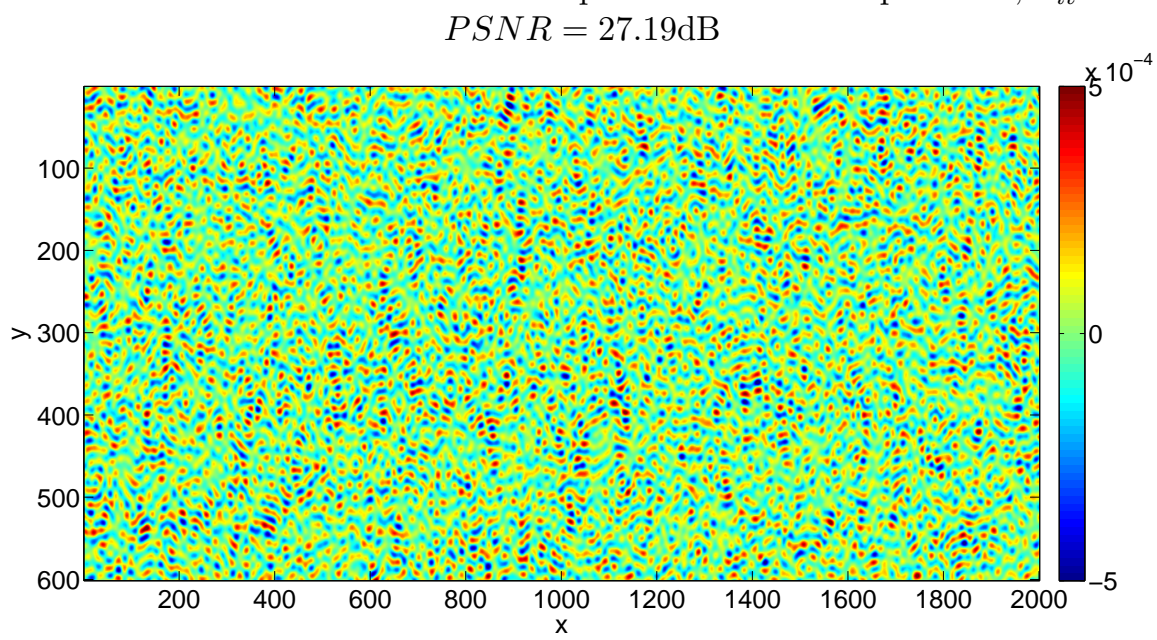

c- difference between deconvolved and reference phase derivative maps. RL $1, n_{i t}=500$ iterations. $P S N R=17.37 \mathrm{~dB}$

Figure 12: Errors maps obtained with RL 1, noise amplitude $N=3, \sigma=8$ pixels 


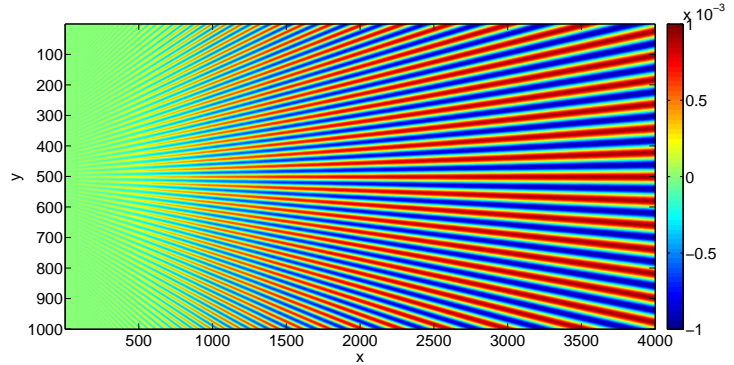

a- Estimated phase derivatives, no noise, $\sigma=5$ pixels

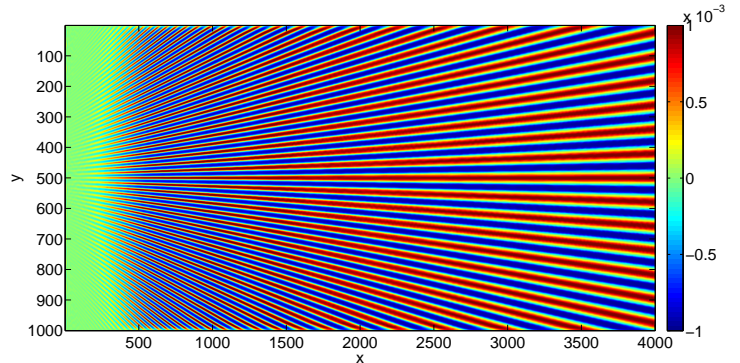

c- Deconvolved phase derivatives, $N=5, \sigma=5$ pixels RL 1, no noise

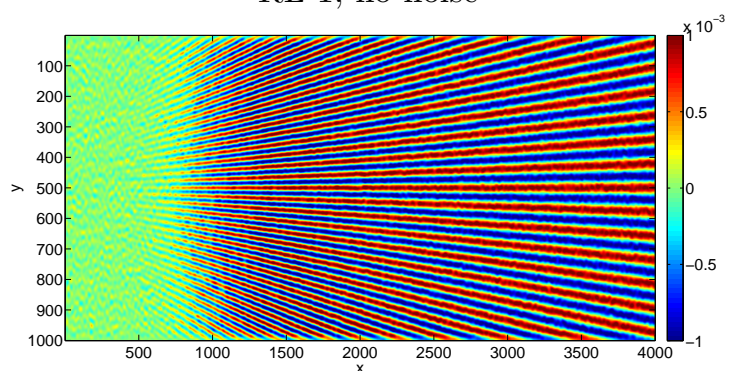

e- Deconvolved phase derivatives RL $1, N=5, \sigma=8$ pixels

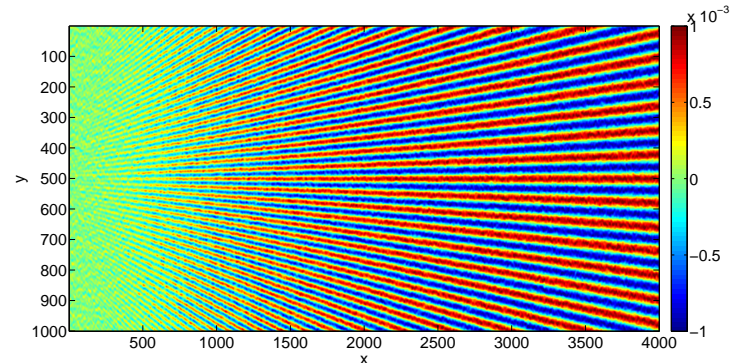

b- Estimated phase derivatives, $N=5, \sigma=5$ pixels

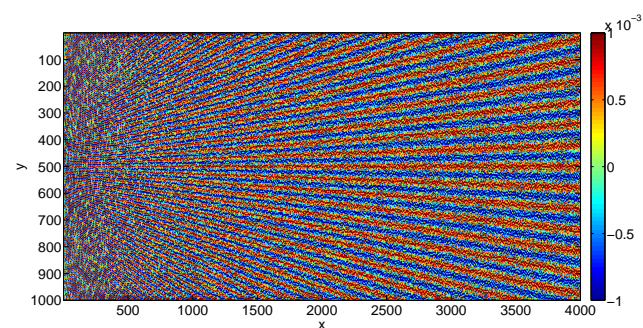

d- Deconvolved phase derivatives, $\mathrm{W} 2, N=5, \sigma=5$ pixels

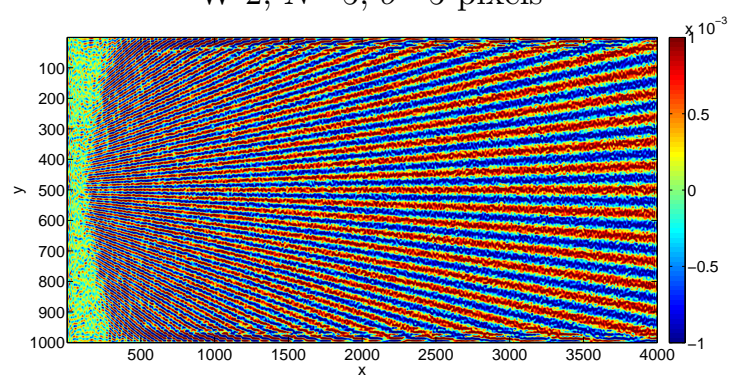

f- Deconvolved phase derivatives $\mathrm{W} 2, N=5, \sigma=8$ pixels

Figure 13: Phase derivatives: comparison between estimated and deconvolved phase derivatives 


\section{Examples}

\subsection{Introduction}

The objective here is to briefly illustrate the effect of the procedure described above on actual strain maps obtained with the grid method. Two short examples have been chosen for this purpose. They are extracted from recent studies in which the grid method was employed to obtain the strain field during mechanical tests. It must however be pointed out that deconvolution was not an objective as these studies were undertaken, so some information is not available here, especially concerning noise. In addition, parameter $\sigma$ used to obtain the maps was not always optimal for deconvolution purposes. Nevertheless the authors think these examples still give a first idea on the strengths and current limitations of this approach.

\subsection{Example 1: strain field on asphalt specimens}

In this example addressed in full details in [30], asphalt specimens were subjected to various compressive tests and the strain fields were measured on one of their faces. Asphalt is mainly made up of aggregates bound by a bitumen matrix. The problem here is the fact that aggregates are separated by narrow bands of binder in some zones (see Figure 14-a) whose width can be lower than one millimeter. The pitch of the grid bonded on the surface is equal to $0.2 \mathrm{~mm}$. Assuming that 5 pixels/period are used to encode the grid image (this choice is a trade-off between spatial resolution, resolution and size of the field under interest), 25 pixels are used to capture the details of the strain field over a distance equal to one millimeter. The Gaussian envelope used to calculate the phases and their derivatives generally exhibits a standard deviation equal at least to $\sigma=5$ pixels to limit the effect of noise [17], so the "width" of the Gaussian envelope is equal to 30 pixels $(6 \times \sigma)$ in this case. This width is greater than the size of the binder band under investigation is these zones (this is worse if $\sigma>5$ pixels), so the procedure for extracting the phase derivatives from the grid images is expected to directly influence the strain level measured within the band.

Figure 14-b shows a typical $\varepsilon_{x x}$ strain map obtained with $\sigma=5$ pixels during a compression test. This image (denoted Image 1 in the following), is obtained at an advanced stage of the test. Hence the strain level in the binder bands reaches up to more than one percent, which is quite significant. Noise influence due to the camera sensor is expected to be limited here, so this situation is a priori favorable for deconvolution. The shape of the bigger aggregates is recognizable in this figure. The observed pattern is consistent with the picture shown in Figure 14-a because the strain level is nearly null in the aggregates compared to that in the binder bands, bitumen being much softer than aggregates.

We focus now as an example on the zone defined by the small black rectangle. An enlargement is shown in Figure 15-a. On close comparison between the width of the apparent band in Figure 14-b and the corresponding zone in the picture shown in Figure 14-a, it clearly appears that the first one is greater, thus illustrating the effect of blurring and meaning that the strain level displayed here is lower than the actual one.

The W 2 and LR 1 methods described and discussed above have been applied in this case. For the first technique, the noise amplitude of the CCD chip was not measured and characterized here. It is however one of the input data of the Wiener filter [18], it is roughly assessed from the data sheet of the camera supplier. For the second technique, the grid image has been processed with $n_{i t}=10$, as suggested in Section 3.5 above. In both cases, $\sigma$ is first chosen to be $\sigma=8$ pixels instead of 5 , as suggested above. The obtained results are shown in Figures 15 -c and -d for W 2 and RL 1, respectively. It can be seen that the strain maps are slightly less blurred than in Figure 14-b, but the strain field seems more noisy over the aggregates, which 
means that noise and other phenomena such as grid defects were not sufficiently erased before deconvolution. Figure 15-b shows the enlarged zone after deconvolution. The same color scale is used for the maps obtained before and after deconvolution (Figure 15-a and -b, respectively). As may be seen, the band seems to be thinner and the strain amplitude at the center is greater, as expected.

To make things clearer, a cross section along the line superimposed in Figure 15-a is shown in Figure 16-a along with the curve obtained with RL 1 (the abscissa measured along this line is denoted $u$ ). Interestingly, the profile is similar to that obtained with $\mathrm{W} 2$. This shows that the results obtained with both methods are consistent. A strain level enhancement clearly appears: the profile is thinner at the base of the distribution and the maximum value reached at the center is now greater. The strain value at the center is greater after deconvolution: $30 \%$ with RL 2 and $33 \%$ with W 2. This illustrates the fact that deconvolution is vital if a quantitative information and not only a qualitative one is sought in this zone.

$\sigma=8$ pixels being expected to be optimal, $\sigma=5$ pixels has been tested to visualize the effect of this parameter. The obtained results are shown in Figures 15-e and -f for $\mathrm{W} 2$ and RL 1, respectively. As may be seen, the corresponding maps are noisier, thus confirming that an optimal value for $\sigma$ exists. The strain profile is shown in Figure 16-b. The initial profile is now higher than with $\sigma=8$ pixels, as expected since blurring is lower, but the restored profiles are noisier merely because the initial profile is also noisier. It is however worth noting that the restored profiles feature nearly the same maximum value as with $\sigma=5$ pixels.

A second image obtained during the same test has also been investigated. It is referred to as Image 2 in the following. It has been chosen at the very beginning of the test, for a much lower load level to examine what happens when the strain level is lower, and therefore the impact of noise greater. Obtained results are shown in Figures 17-a -b. As may be seen, some significant blobs appear. They are due to noise and some other phenomena such as grid defects which were not sufficiently erased in the strain map before deconvolution and/or, concerning the Wiener filter, too rough a characterization of noise. The band profile obtained after deconvolution with $\sigma=8$ pixels is shown in Figure 16-c. The impact of noise is visible. Interestingly, the magnitude of the restoration is comparable to that observed for Image 1 above, but it is worth noting that we have here a strain level which is approximately ten times lower (compare the strain levels in Figures 16-b and -c). This suggests that the "degree of restoration" mainly depends on the geometry of the specimen, in other words the actual thickness of the band in the current example. Finally, Figure 16-d shows what happens when $\sigma=5$ pixels: the impact of noise becomes now too significant and the information is lost.

In conclusion, it can be said that results obtained here are promising. Keeping in mind that noise was not properly characterized here, there is room for further improvements. In particular, it is shown in [18] that the phase derivative variance can be predicted if the noise variance of the CCD chip is know. This result has been used in the simulations discussed in Section 3.3 above. This is however not enough when actual grids are considered: they feature indeed some pitch variations or some local lack of ink which should be characterized specifically. The lens also induces a PSF which certainly influences the strain maps. Tackling these issues is however beyond the scope of the current paper.

\subsection{Example 2: strain field on a shape memory alloy}

This second example deals with a tensile test performed on a specimen of monocrystalline CuAlBe shape memory alloy. It is austenite at room temperature, so when a tensile test is performed, martensite bands or needles suddenly appear and propagate throughout the specimen, as discussed in [31]. These bands are generally very thin when they appear, so only slight 


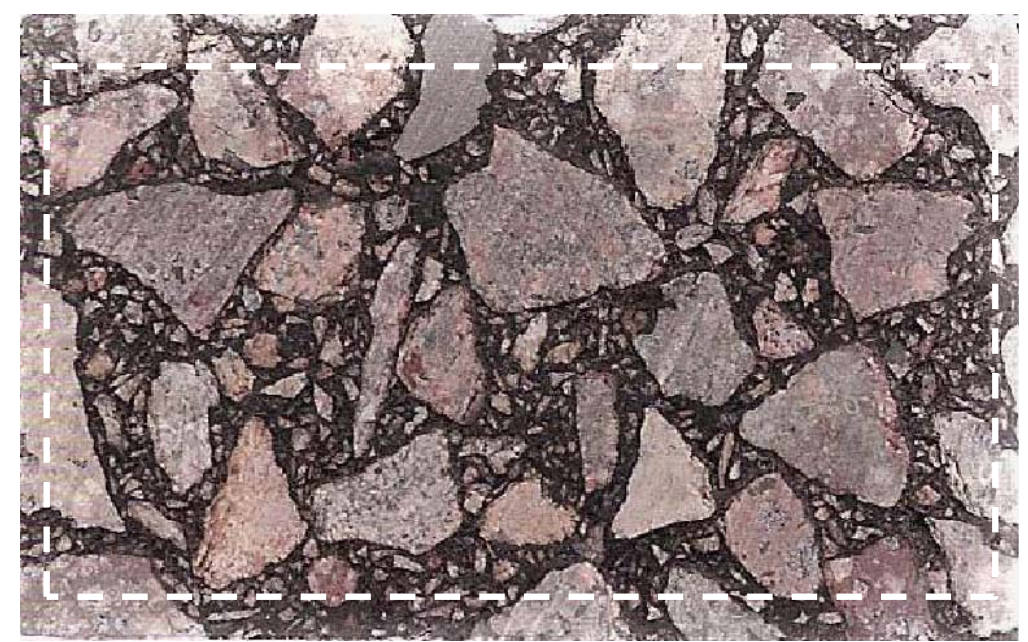

a- Front face of the specimen

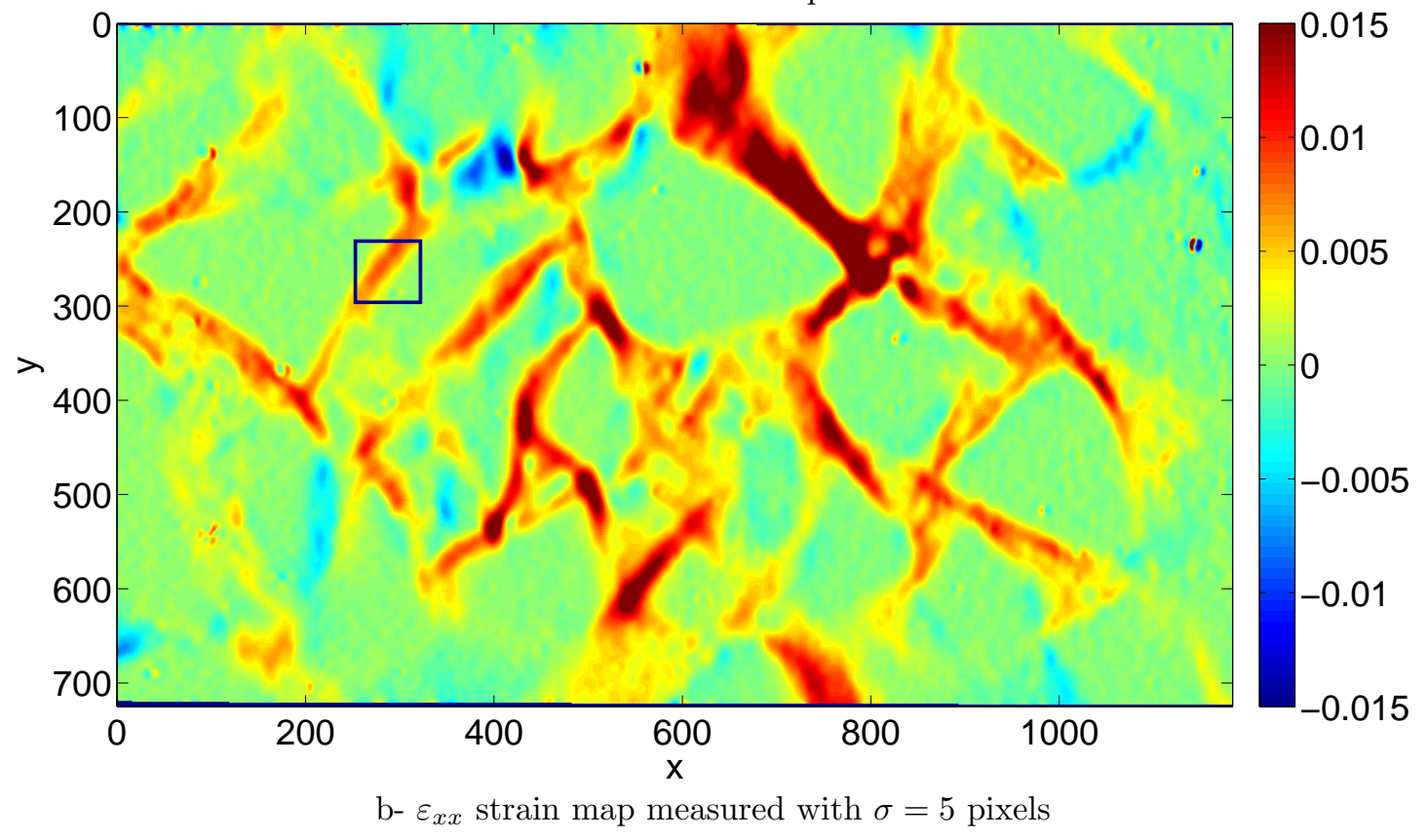

Figure 14: Asphalt specimen under compression [30] 

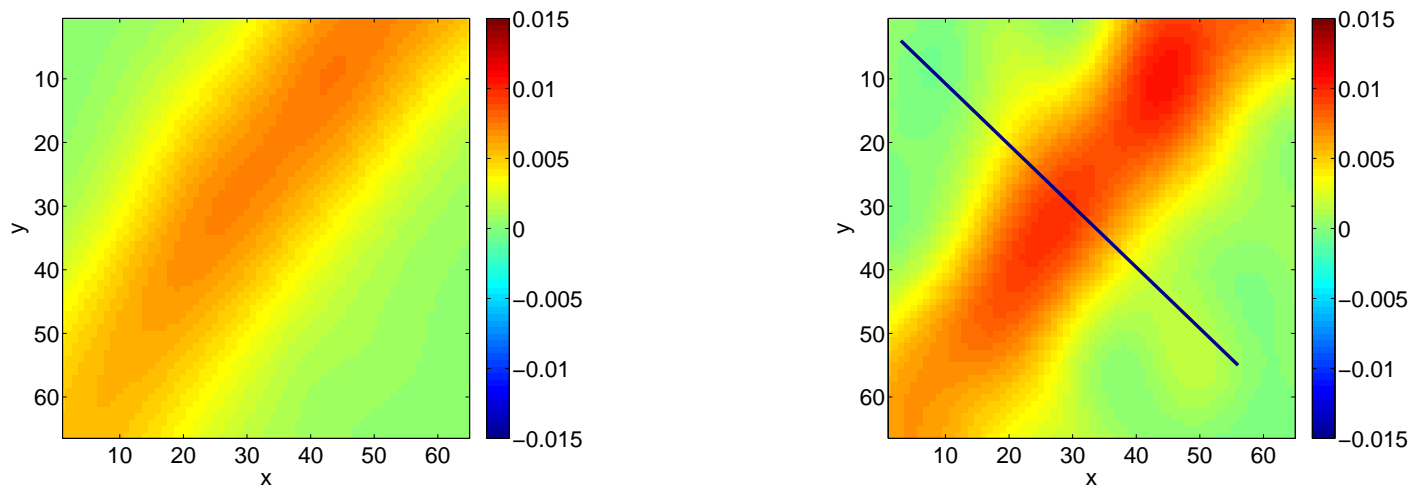

a- Enlargement of Zone A (initial, $\sigma=8$ pixels), Image 1

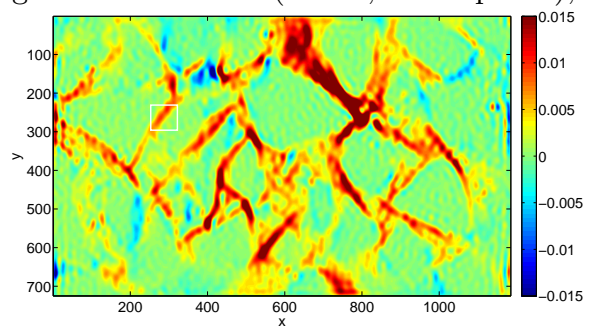

b- Enlargement of Zone A (W2, $\sigma=8$ pixels), Image 1

c- Image 1 deconvolved with $\mathrm{W} 2, \sigma=8$ pixels

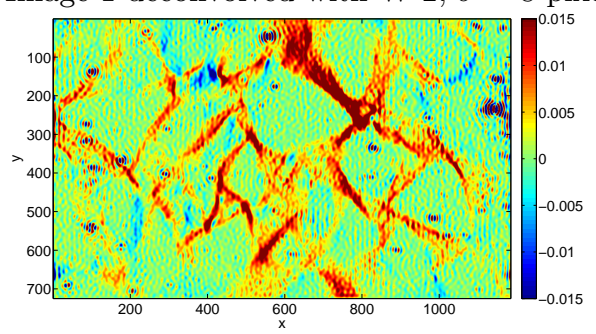

e- Image 1 deconvolved with $\mathrm{W} 2, \sigma=8$ pixels

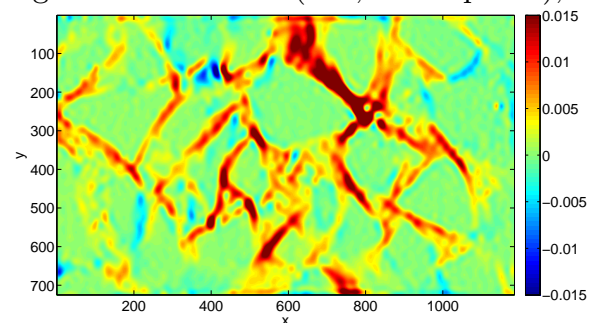

d- Image 1 deconvolved with RL $1, \sigma=8$ pixels

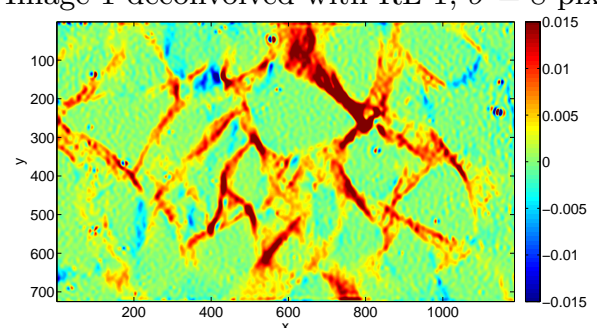

f- Image 1 deconvolved with RL $1, \sigma=5$ pixels

Figure 15: Asphalt specimen under compression. Deconvolution of Image 1 for load level 1 


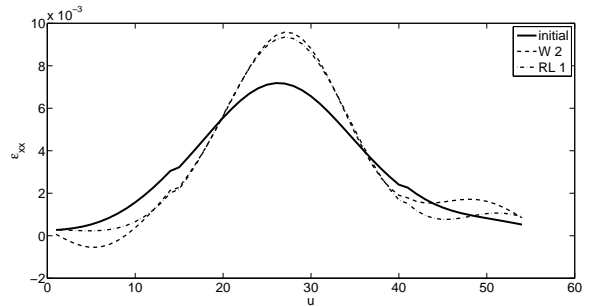

a- cross section of Zone A ( $\sigma=8$ pixels), Image 1

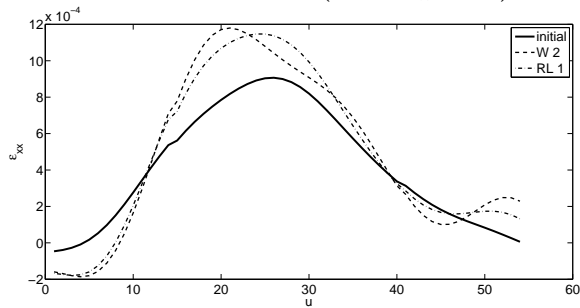

c- cross section of Zone A ( $\sigma=8$ pixels), Image 2

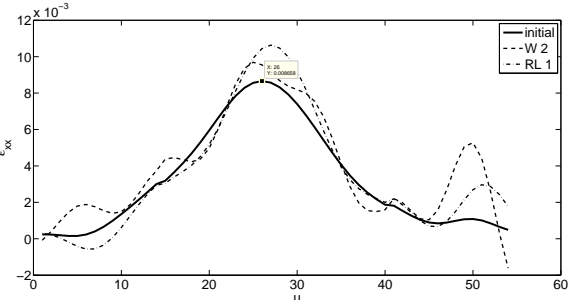

b- cross section of Zone A ( $\sigma=5$ pixels), Image 1

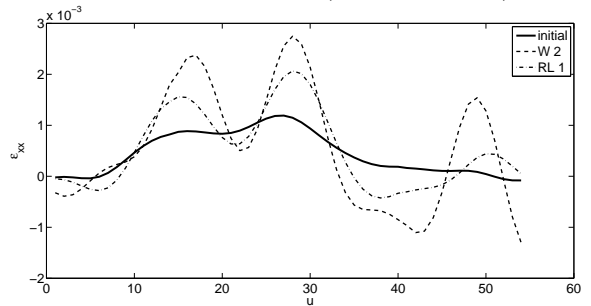

d- cross section of Zone A ( $\sigma=5$ pixels), Image 2

Figure 16: Asphalt specimen under compression. Cross section of the binder band in Zone A

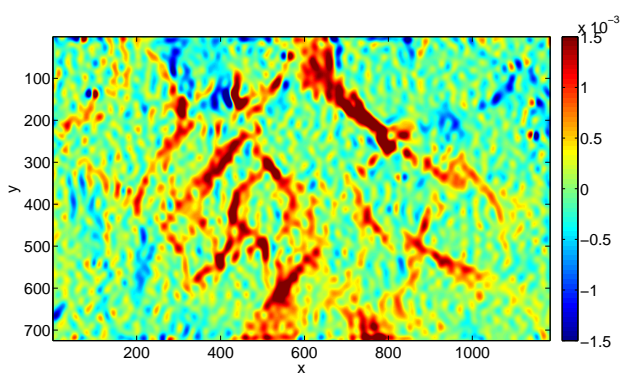

a- Deconvolved image 2. RL $1, \sigma=8$ pixels

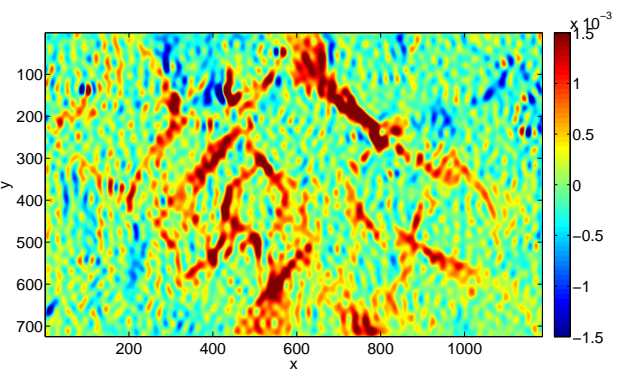

b- Deconvolved image 2. W 2, $\sigma=8$ pixels

Figure 17: Deconvolution of Image 2 
bands are visible in the strain field. It must be pointed out that this case is very favorable for deconvolution since the strain amplitude within these bands is immediately very high (some percents) because they are due to a phase change, so the noise amplitude is very limited compared to the measured strain thanks to the width of the kernel used in [31] to process the grid images: $\sigma=5$ pixels.

A typical Green-Lagrange $E_{y y}$ strain field (obtained $i$ - by calculating the Hencky strain tensor by adding small strain increments and then $i i$ - by deducing directly the Green-Lagrange tensor) is shown in Figure 18-a, where $y$ represents the vertical direction along which the tensile force is applied. The blue color corresponds to austenitic regions (only very slightly elastically stretched during the test) whereas the red color corresponds to martensitic regions since the phase change gives rise to a sudden and significant deformation.

In the white rectangle enlarged in Figure 19-a, it is clear that a hell blue band containing some slight streaks has appeared. The color is not the same as that obtained in wider bands located slightly higher. It can be checked that later on, during the same test, this band becomes progressively wider and reaches the same color as that observed above in the specimen [31].

The width of the band in the white rectangle being lower than the width of the Gaussian envelope used for processing the grid images (a circle featuring a $6 \times \sigma$ diameter is superimposed to the map), one can reasonably guess that the actual strain level in this band is greater than the apparent one. Applying here the RL 2 method reveals that two needles and not only one were hidden behind the unique blurred band in Figure 19-a, which could not really be guessed. In addition, the strain level in these needles is very similar to that observed above in the figure (see Figure 19-b), where austenite has already transformed into martensite. This is quite logical since it means that the martensite variant obtained in needles is the same as that obtained above in wider bands, as expected. Figure 20 finally shows the strain distribution along the white portion of line superimposed to the preceding figure. Strain enhancement clearly appears. It can be seen that the thickness of the needles (estimated at half the maximum), is equal to about 10 pixels, which is equal here to $0.4 \mathrm{~mm}$, the size of the pixels being equal to 40 micrometers in this case. 


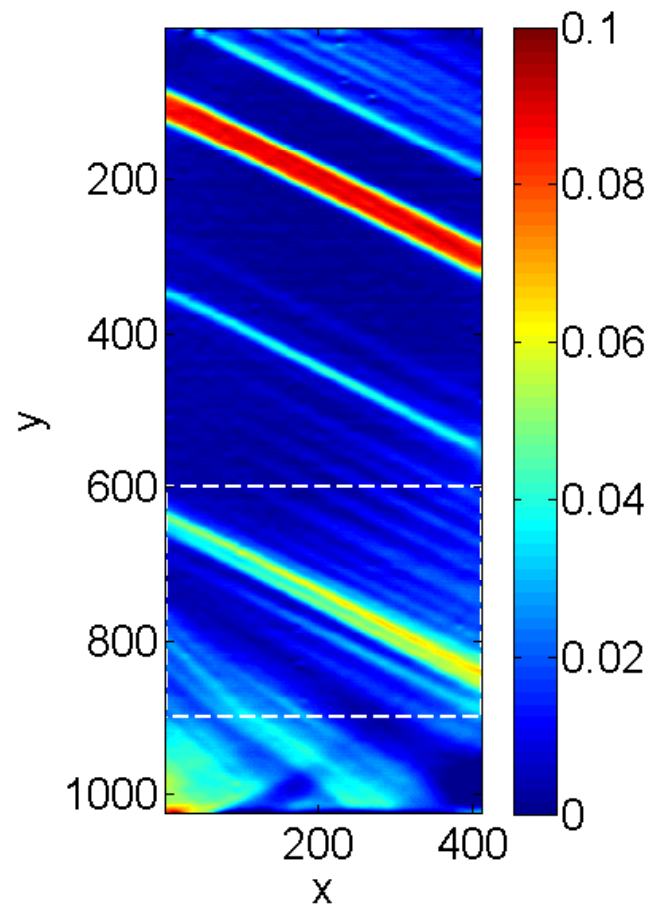

a- Before deconvolution [31]

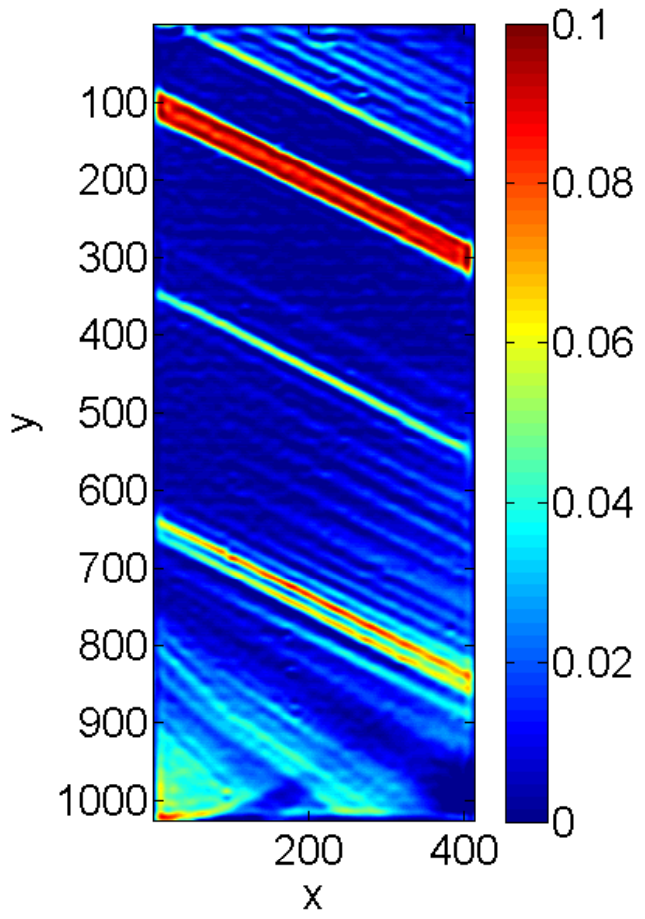

b- After deconvolution

Figure 18: Shape memory alloy under tension. $E_{y y}$ map [31] 


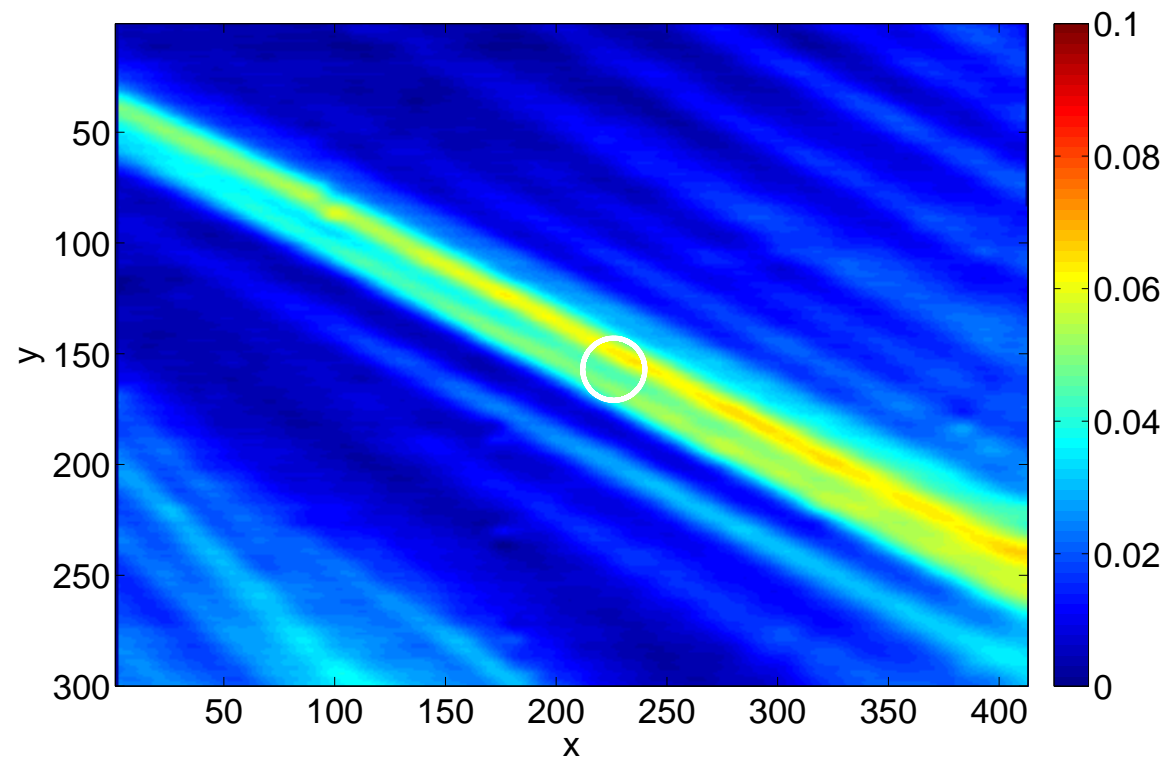

a- Before deconvolution

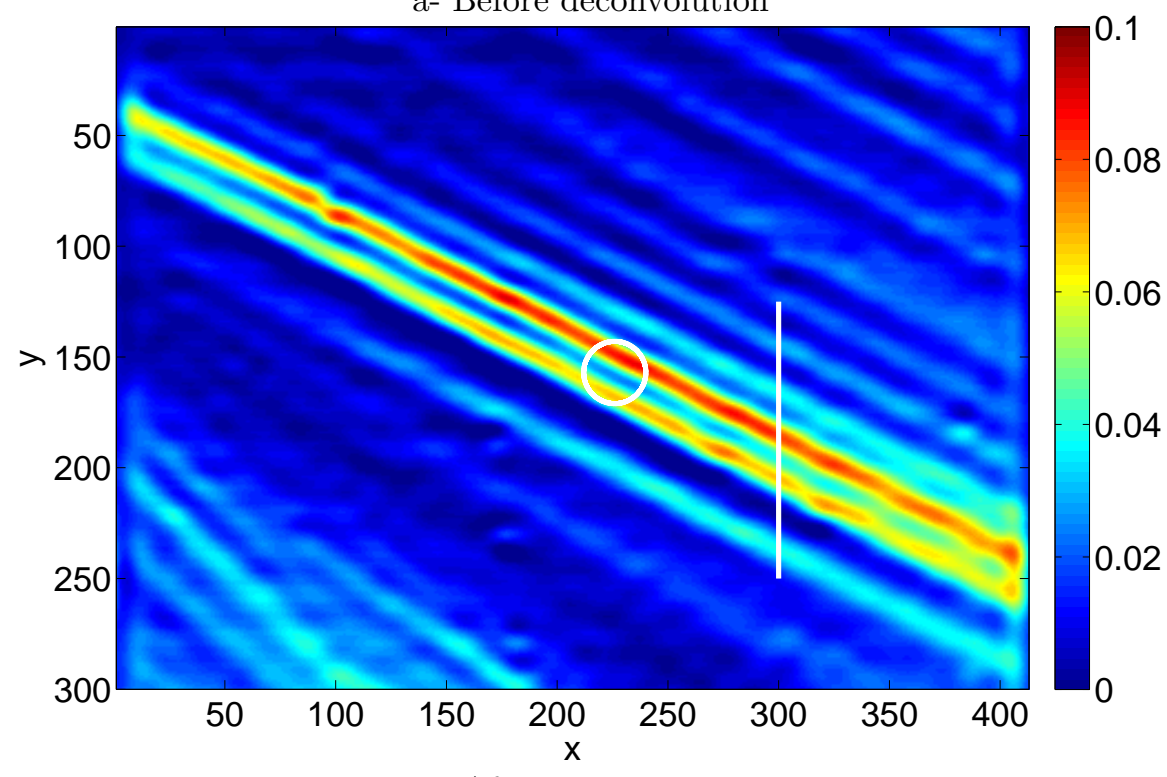

b- After deconvolution

Figure 19: Shape memory alloy under tension. Zoom of the white rectangle 


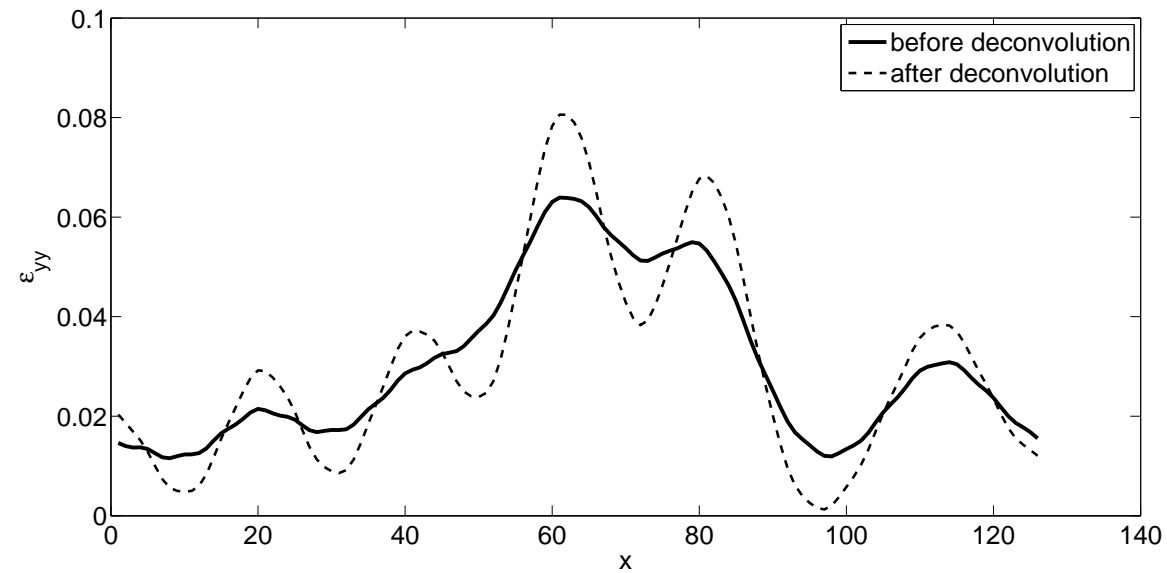

Figure 20: Shape memory alloy under tension. Cross section of the strain distribution 


\section{Conclusion}

In this paper, various deconvolution techniques are presented and used to restore strain fields obtained with the grid method. A synthetic example serves as a common thread between various numerical examples which enable to assess the performance of these deconvolution techniques. The influence of noise on the quality of the deconvolved maps is a major issue since some techniques are not robust whereas some other ones still give good results when noise corrupt grid images. Thanks to the regular marking, it is possible to establish a link between noise variance in the camera sensor and strain variance. Simulations have shown that taking this information in the Wiener filter led to the best results if noise is correctly characterized. Two examples of actual strain fields were also studied. In these cases, it is clear that some additional phenomena such as slight grid pitch variations, local grid bonding defects or PSF of the optical device itself corrupt the actual strain fields when the signal to noise ratio decreases. These phenomena could not be taken into account in the current work. Characterizing them separately should be undertaken to provide a more complete and realistic information on the deterioration of the final strain maps. This should then improve actual image enhancement procedures based on deconvolution. Another extension could be to deconvolve simultaneously the three in-plane strain components under the constraints of the compatibility equations which link the second-derivatives of the strain components.

\section{Acknowledgements}

Dr D. Delpueyo, Prof. X. Balandraud and Prof. E. Toussaint are gratefully acknowledged for fruitful discussions about the experimental results shown in the two examples. 


\section{References}

[1] D. Lecompte, A. Smits, S. Bossuyt, H. Sol, J. Vantomme, D. Van Hemelrijck, and A.M. Habraken. Quality assessment of speckle patterns for digital image correlation. Optics and Lasers in Engineering, 44:1132-1145, 2006.

[2] B. Pan, Z. Lu, and H. Xie. Mean intensity gradient: an effective global parameter for quality assessment of the speckle patterns used in digital image correlation. Optics and Lasers in Engineering, 48:469-477, 2010.

[3] Y. Barranger, P. Doumalin, J.-C. Dupré, and A. Germaneau. Strain measurement by digital image correlation: influence of two types of speckle patterns made from rigid or deformable marks. Strain, 48(5):357-365, 2012.

[4] M. Bornert, F. Brémand, P. Doumalin, J.-C. Dupré, M. Fazzini, M. Grédiac, F. Hild, S. Mistou, J. Molimard, J.-J. Orteu, L. Robert, Y. Surrel, P. Vacher, and B. Wattrisse. Assessment of digital image correlation measurement errors: methodology and results. Experimental Mechanics, 49(3):353-370, 2009.

[5] P. Lava, S. Cooreman, S. Coppieters, M. De Strycker, and D. Debruyne. Assessment of measuring errors in DIC using deformation fields generated by plastic FEA. Optics and Lasers in Engineering, 47:747-753, 2009.

[6] Y. Q. Wang, M.A. Sutton, H.A. Bruck, and H.W. Schreier. Quantitative error assessment in pattern matching: effects of intensity pattern noise, interpolation, strain and image contrast on motion measurements. Strain, 45:160-178, 2009.

[7] L.B. Meng, G.C. Jin, and X.F. Yao. Application of iteration and finite element smoothing technique for displacement and strain measurement of digital speckle correlation. Optics and Lasers in Engineering, 45(1):57-63, 2007.

[8] S. Avril, P. Feissel, F. Pierron, and P. Villon. Comparison of two approaches for controlling the uncertainty in data differentiation: application to full-field measurements in solid mechanics. Measurement Science and Technology, 21(1):15703-15713, 2007.

[9] E. A. Patterson, E. Hack, P. Brailly, R. L. Burguete, Q. Saleem, T. Siebert, R. A. Tomlinson, and M. P. Whelan. Calibration and evaluation of optical systems for full-field strain measurement. Optics and Lasers in Engineering, 45(5):550-564, 2007.

[10] H. Haddadi and S. Belhabib. Use of a rigid-body motion for the investigation and estimation of the measurement errors related to digital image correlation technique. Optics and Lasers in Engineering, 46:185-196, 2008.

[11] C. Badulescu, M. Bornert, J.-C. Dupré, S. Equis, M. Grédiac, J. Molimard, P. Picart, R. Rotinat, and V. Valle. Demodulation of spatial carrier images: performance analysis of several algorithms using a single image. Experimental Mechanics, 2013. In press.

[12] R.C. Gonzalez and R.E. Woods. Digital Image Processing (3rd Edition). Prentice-Hall, 2006.

[13] Y. Surrel. Photomechanics, Topics in Applied Physic 77, chapter Fringe Analysis, pages 55-102. Springer, 2000. 
[14] P. A. Gorry. General least-squares smoothing and differentiation by the convolution (Savitzky-Golay) method. Anal. Chem., 62:570-573, 1990.

[15] P. Feissel. From displacements to strains. In M. Grédiac and F. Hild, editors, Full-Field measurements and identification in solid mechanics. Wiley, 2013.

[16] C. Badulescu, M. Grédiac, J.-D. Mathias, and D. Roux. A procedure for accurate one-dimensional strain measurement using the grid method. Experimental Mechanics, 49(6):841-854, 2009.

[17] C. Badulescu, M. Grédiac, and J.-D. Mathias. Investigation of the grid method for accurate in-plane strain measurement. Measurement Science and Technology, 20(9):20:095102, 2009.

[18] F. Sur and M. Grédiac. Towards deconvolution to enhance the grid method for in-plane strain measurement. Under review.

[19] A. M. Maas and P. A. A. M. Somers. Two-dimensional deconvolution applied to phasestepped shearography. Optics and Lasers in Engineering, 26:1594-1602, 2006.

[20] B. Dhanasekar and B.Ramamoorthy. Restoration of blurred images for surface roughness evaluation using machine vision. Tribology International, 43:268-276, 2010.

[21] A. Delaiglea and P. Hallb. On optimal kernel choice for deconvolution. Statistics and Probability Letters, 76:1594-1602, 2006.

[22] S. Avril, P. Feissel, F. Pierron, and P. Villon. Estimation of the strain field from full-field displacement noisy data. European Journal of Computational Mechanics, 17(5-7):857-868, 2008.

[23] J. L. Starck, E. Pantin, and F. Murtagh. Deconvolution in astronomy: A review. Publications of the Astronomical Society of the Pacific, 114(800):1051-1069, 2002.

[24] J. Aelterman, B. Goossens, A. Pizurica, and W. Philips. Suppression of correlated noise, pages 211-236. Recent advances in signal processing. In-Tech, 2010.

[25] A.N. Tikhonov and V.A. Arsenin. Solutions of ill-posed problems. Winston and Sons, 1977.

[26] W. H. Richardson. Bayesian-based iterative method of image restoration. Journal of the Optical Society of America, 62(1):55-59, 1972.

[27] L. B. Lucy. An iterative technique for the rectification of observed distributions. Astronomical Journal, 79(6):745-754, 1974.

[28] N. Wiener. Extrapolation, Interpolation, and Smoothing of Stationary Time Series. The MIT Press, 1964.

[29] Matlab. http://www.mathworks.fr/fr/help/images/image-restoration-deblurring.html, 2012.

[30] M. Grédiac and E. Toussaint. Studying the mechanical behaviour of asphalt mixtures with the grid method. Strain. An International Journal for Experimental Mechanics, 49(1): 1-15, 2013. Wiley.

[31] D. Delpueyo, M. Grédiac, X. Balandraud, and C. Badulescu. Investigation of martensitic microstructures in a monocrystalline $\mathrm{Cu}$-Al-Be shape memory alloy with the grid method and infrared thermography. Mechanics of Materials, 45:34-51, 2012. Elsevier. 